Pacific Journal of Mathematics

TRIPLES OF OPERATOR-VALUED FUNCTIONS RELATED TO 


\section{TRIPLES OF OPERATOR-VALUED FUNCTIONS RELATED TO THE UNIT CIRCLE}

\section{A. G. GIBSON}

In this paper various triples of operator-valued functions acting in a Hilbert space are characterized, and the members are shown to be connected by a one-to-one-to-one correspondence. The elements of the triples are operator measures, generalized resolvents, and positive definite sequences which are related to the unit circle. The relationships between operator measures and positive definite sequences were first obtained by M. A. Naimark and B.Sz.-Nagy in their dilation and moment theorems. The main contribution of this paper is a characterization of the interrelated resolvent classes. By exploiting the correspondence between the various classes, a unified development of the theory is obtained.

R. McKelvey [8] developed the interrelations among three classes of operator-valued functions $\left\{E_{t}, R_{\lambda}, V_{s}\right\}$ related to operators with spectrum in a half-plane. In the prototype for the general situation these functions were associated with a self-adjoint operator $T$, and $E_{t}$ was its spectral function, $R_{\lambda}$ its resolvent, and $V_{s}$ the unitary group $e^{-i s T}$. In more general cases, the three classes were associated with dissipative and symmetric operators.

In this study we investigate the interrelations between three analogous classes of operator-valued functions $\left\{E_{\theta}, R_{z}, T^{(k)}\right\}$ related to operators with spectrum in the unit circle. In the prototype these functions are associated with a unitary operator $T$, and $E_{\theta}$ is its spectral function, $R_{z}$ its resolvent, and $T^{(k)}$ the cyclic group of its integral powers. Generalizations here include the triples associated with contraction, isometric, and partially unitary operators. In our most general case, the triple $\left\{E_{\theta}, R_{z}, T^{(k)}\right\}$ belongs to $\mathscr{S}=\{\mathscr{E}, \mathscr{R}, \mathscr{T}\}$, where the classes in the triple $\mathscr{S}$ are called operator distribution functions, generalized resolvents, and positive definite operator-valued sequences respectively. In saying that the triple of operator-valued functions $\left\{E_{\theta}, R_{z}, T^{(k)}\right\}$ belongs to the triple of classes $\mathscr{S}=\{\mathscr{E}, \mathscr{R}, \mathscr{T}\}$ we shall mean $E_{\theta} \in \mathscr{E}, R_{z} \in \mathscr{R}$, and $T^{(k)} \in \mathscr{T}$, i.e., a triple of classes is to be understood in the dual sense of a triple and as the Cartesian product of the classes $\mathscr{E}, \mathscr{R}, \mathscr{T}$.

The classes $\mathscr{E}, \mathscr{R}$, and $\mathscr{T}$ of bounded linear operators on the complex Hilbert space $\mathscr{H}$ to $\mathscr{C}$ are defined as follows:

( $\alpha) \quad E_{\theta}(0 \leqq \theta \leqq 2 \pi)$ belongs to the class $\mathscr{E}=\mathscr{E}(\mathscr{C})$ whenever

(a) $0 \leqq\left(E_{\theta_{1}} x, x\right) \leqq\left(E_{\theta_{2}} x, x\right) \leqq(x, x)$ for $0 \leqq \theta_{1}<\theta_{2} \leqq 2 \pi$ and 

$x \in \mathscr{H}$.
(b) $E_{\theta}=E_{\theta+0} \equiv \operatorname{Lim}_{\varphi \rightarrow \theta^{+}} E_{\varphi}, 0 \leqq \theta<2 \pi$.
(c) $E_{0}=0$.

( $\beta) \quad R_{z}(|z| \neq 1)$ belongs to the class $\mathscr{R}=\mathscr{R}(\mathscr{H})$ whenever

(a) $R_{z}$ is a holomorphic function of $z$ for $|z| \neq 1$.

(b)

$$
\left\|R_{z} x\right\|^{2} \leqq \frac{2 \operatorname{Re}\left(R_{z} x, x\right)-\left(R_{0} x, x\right)}{1-|z|^{2}}, \quad|z| \neq 1, x \in \mathscr{C},
$$

i.e., $\left(R_{0} x, x\right)$ is real and this inequality holds.

(c) $R_{z *}=R_{0}-R_{z}^{*}, 0<|z|<1$ or $|z|>1$, where $z^{*}=1 / \bar{z}$.

( $\gamma) \quad T^{(k)}( \pm k=0,1,2, \cdots)$ belongs to the class $\mathscr{T}=\mathscr{T}(\mathscr{C})$ whenever

(a) $T^{(0)} \leqq I$.

(b) The sequence $\left\{T^{(k)}\right\}$ is positive definite, i.e.,

$$
\sum_{j, k=-n}^{n} \sum^{n}\left(T^{(j-k)} x_{j}, x_{k}\right) \geqq 0
$$

for $n=1,2, \cdots$, and arbitrary sequences $\left\{x_{k}\right\}_{-n}^{n}$ of vectors in $\mathscr{H}$.

In $\S 2$ a functional calculus will be presented wich is based on a mapping from a class of functions bounded and measurable with respect to the operator distribution function $E_{\theta} \in \mathscr{E}$ to a class of bounded operators. It is similar to the functional calculus previously developed by M. Schreiber $[12,13]$, and to that known for the unitary operator $[10, \S 109]$. We then prove the main theorem of this paper which is the following:

THeOREM A. Each function $E_{\theta} \in \mathscr{E}, R_{z} \in \mathscr{R}$, or $T^{(k)} \in \mathscr{T}$ belongs to a unique triple of functions $\left\{E_{\theta}, R_{z}, T^{(k)}\right\}$ in $\mathscr{S} \equiv\{\mathscr{E}, \mathscr{R}, \mathscr{T}\}$, such that the members of the triple are interrelated by formulas (1)-(6).

Formulas (1)-(6) are the following representations which will be seen in $\S 2.1$ to make sense in either the strong or weak topology.

$$
\begin{aligned}
R_{z} & =\int_{0}^{2 \pi} \frac{1}{1-e^{i \theta} z} d E_{\theta} \quad(|z| \neq 1) . \\
T^{(k)} & =\int_{0}^{2 \pi} e^{i k \theta} d E_{\theta} \quad( \pm k=0,1,2, \cdots) . \\
R_{z} & =\sum_{k=0}^{\infty} z^{k} T^{(k)} \quad(|z|<1) \\
& =-\sum_{k=1}^{\infty} z^{-k} T^{(-k)} \quad(|z|>1) .
\end{aligned}
$$


(4)

$$
\begin{aligned}
E_{\theta_{2}}-E_{\theta_{1}}=\frac{1}{\pi} \operatorname{Lim}_{r-1^{-}} & \int_{\theta_{1}}^{\theta_{2}} \operatorname{Re} R_{r \exp (-i \theta)} d \theta-\frac{\left(\theta_{2}-\theta_{1}\right)}{2 \pi} R_{0} \\
& \left(\theta_{1}, \theta_{2} \text { points of continuity of } E_{\theta}\right) .
\end{aligned}
$$

$$
\begin{aligned}
E_{\theta_{2}}-E_{\theta_{1}}=\frac{1}{2 \pi} \sum_{-\infty}^{\infty} \frac{e^{-i k \theta_{1}}-e^{-i k \theta_{2}}}{i k} T^{(k)} & \left(\theta_{1}, \theta_{2} \text { points of continuity of } E_{\theta}\right) \text {. }
\end{aligned}
$$

$$
\begin{aligned}
T^{(k)} & =\left.\frac{d^{k} R_{z}}{k ! d z^{k}}\right|_{z=0} \quad(k=0,1,2, \cdots) \\
T^{(-k)} & =-\frac{1}{k !} \operatorname{Lim}_{z \rightarrow 0} \frac{d^{k} R_{1 / z}}{d z^{k}} \quad(k=1,2, \cdots) .
\end{aligned}
$$

Definition. A triple of functions $\left\{E_{\theta}, R_{z}, T^{(k)}\right\} \in \mathscr{S}$, whose members are related as in Theorem $\mathrm{A}$, will be called a matched triple of functions.

The new parts of Theorem A are the characterization $(\beta)$ of the class $\mathscr{R}$, and the inversion formulas (4) and (5). The relationship between $\mathscr{E}$ and $\mathscr{T}$ is essentially a generalization of Naimmarks moment theorem obtained by S. K. Berberian in [3, Ths. 3,4]. In Theorem B it is shown that those generalized resolvents $R_{z} \in \mathscr{R}$ which satisfy the additional condition $R_{0}=I$ can be represented as the ordinary resolvent of a variable contraction operator $T_{z}$ on $\mathscr{K}$ which is a holomorphic function of $z$ for $|z|<1$.

Definition. A triple of classes $\mathscr{S}^{\prime}=\left\{\mathscr{C}^{\prime}, \mathscr{R}^{\prime}, \mathscr{T}^{\prime}\right\}$ will be called a matched triple of classes if and only if

(i) $\mathscr{S}^{\prime} \cong \mathscr{S}$; i.e., $\mathscr{E}^{\prime} \cong \mathscr{E}, \mathscr{R}^{\prime} \subseteq \mathscr{R}$ and $\mathscr{T}^{\prime} \cong \mathscr{T}$.

(ii) When $\left\{E_{\theta}, R_{z}, T^{(k)}\right\}$ is a matched triple of functions in $\mathscr{S}$, then any one of the relations $E_{\theta} \in \mathscr{E}^{\prime}, R_{z} \in \mathscr{R}^{\prime}, T^{(k)} \in \mathscr{T}^{\prime}$ implies all three, i.e., implies that the triple is in $\mathscr{S}^{\prime}$.

A characterization of the matched triple of classes $\mathscr{S}_{0}=\left\{\mathscr{E}_{0}, \mathscr{R}_{0}, \mathscr{T}_{0}\right\}$ associated with a unitary operator is given in $\S 4$. Also, we shall develop the matched triple of classes $\mathscr{S}_{1}=\left\{\mathscr{E}_{1}, \mathscr{R}_{1}, \mathscr{T}_{1}\right\}$ related to a partially unitary operator (an operator which can be written as the direct sum of a unitary operator and the zero operator). It will be shown that every matched triple of functions in $\mathscr{S}(\mathscr{C})$ is a projection of a matched triple of functions in $\mathscr{S}_{1}\left(\mathscr{Y}^{+}\right)$, where $\mathscr{K}^{+}$is a Hilbert space extension of $\mathscr{C}$.

In $\S 5$ we prove a characterization of the matched triple of classes $\mathscr{S}_{2}=\left\{\mathscr{E}_{2}, \mathscr{R}_{2}, \mathscr{T}_{2}\right\}$ associated with a contraction operator on $\mathscr{C}$, and show that a multiplicative functional calculus may be obtained in this case. The relationship between the classes $\mathscr{E}_{2}$ and $\mathscr{T}_{2}$ was first 
established by Sz.-Nagy [17] and M. Schreiber [12]. The characterization of the interrelated class $\mathscr{R}_{2}$ appears to be new.

The isometric operator case is discussed in $\S 6$. Equivalent characterizations of the class of generalized resolvents of an isometric operator are obtained, and Theorem B is used to obtain a new characterization which leads to a simple and direct proof of a formula for the form of all generalized resolvents of a closed isometric operator.

\section{The general theory of the triple $\mathscr{S}$.}

2.1. A study of the relationships between the classes $\mathscr{E}, \mathscr{R}$, and $\mathscr{T}$ depends in large part upon the exploitation of a functional calculus analogous to that for a unitary operator [10, $\$ 109]$. We begin by showing that we may integrate a class of complex-valued functions with respect to operator distribution functions $E_{\theta}$.

Suppose $\mathrm{E}_{\theta} \in \mathscr{E}(\mathscr{K})$, i.e., $E_{\theta}$ satisfies the conditions $(\alpha)$ of $\S 1$. If $\Delta$ is the left-open-right-closed interval $\left(\theta_{1}, \theta_{2}\right] \subset[0,2 \pi]$, define

$$
E(\Delta) \equiv E_{\theta_{2}}-E_{\theta_{1}} \text {. }
$$

Since $\left(E_{j} x, x\right)$ is real valued, nondecreasing, and continuous on the right, there exists a unique Borel measure $\mu_{x}$ (fixed $x$ ) such that

$$
\mu_{x}(\Delta)=\left(E_{\theta_{2}} x, x\right)-\left(E_{\theta_{1}} x, x\right)
$$

[11, p. 227]. If $\chi(\sigma ; \theta)$ is the characteristic function of the Borel set $\sigma \subseteq[0,2 \pi]$, then the Borel measure $\mu_{x}$ may be obtained from the cumulative distribution function $\left(E_{0} x, x\right)$ by the formula

$$
\mu_{x}(\sigma)=\int_{0}^{2 \pi} \chi(\sigma ; \theta) d\left(E_{\theta} x, x\right) .
$$

We now consider $\mu_{x}(\sigma)$ as a functional in $x$ with $\sigma$ fixed, and uniquely define another functional dependent on a pair of vectors $x, y, \in \mathscr{X}$ by the polarization formula

$$
\mu_{x, y}(\sigma) \equiv \frac{1}{4}\left[\mu_{x+y}(\sigma)-\mu_{x-y}(\sigma)+i \mu_{x+i y}(\sigma)-i \mu_{x-i y}(\sigma)\right] .
$$

As the bilinear form $\left(E_{v} x, y\right)$ and the quadradic form $\left(E_{\theta} x, x\right)$ are also related by the polarization formula [20, p. 322], it follows that

$$
\mu_{x, y}(\sigma)=\int_{0}^{2 \pi} \chi(\sigma ; \theta) d\left(E_{0} x, y\right) .
$$

It is easy to see that $\mu_{x, y}(\sigma)$ is a symmetric bilinear functional of $x$ and $y$ such that $\left|\mu_{x, x}(\sigma)\right| \leqq\|x\|^{2}$. Therefore, $\mu_{x, y}(\sigma)=(E(\sigma) x, y)$, where $E(\sigma)$ is a uniquely determined linear operator with domain $\mathscr{C}$ and $\|E(\sigma)\| \leqq 1[1, \S 21]$. Also $E(\sigma)$ is countable additive in the 
strong or weak operator topology, and $E([0,2 \pi])=\mathrm{E}_{2 \pi} \leqq I$ (cf. [12, p. 580]). $E(\sigma)$ is called an operator measure. M. Schreiber considered the case $E_{2 \pi}=I[12,13]$, and S. K. Berberian [3] considered nonnormalized operator measures.

Operator measures are a generalization of the concept of spectral measures or resolutions of the identity associated with normal operators to the case where the orthogonality condition $E(\sigma)^{2}=E(\sigma)$ is not required. Since $E_{\theta} \in \mathscr{E}$ is the cumulative distribution for the operator measure $E(\sigma)$, and one determines the other, we call the class $\mathscr{E}$ the class of operator distribution functions. The support of $E(\sigma)$, denoted $\Lambda(E)$, is the complement of the union of all open sets where $E(\sigma)$ vanishes.

Denote by $f\left(e^{i \theta}\right), 0 \leqq \theta \leqq 2 \pi$, a complex-valued function which is defined on the unit circle in the complex plane, and denote by $B(d E)$ the class of functions $f\left(e^{i \rho}\right)$ which, when considered as a function of $\theta$, are bounded and Borel measurable on $\Lambda(E)$. It has been shown by M. Schreiber [12, p. 580] that if $f\left(e^{i \rho}\right) \in B(d E)$, then one may define the operator $F$ by the integral

$$
F \equiv \int_{0}^{2 \pi} f\left(e^{i \theta}\right) d E_{\theta}
$$

and this integral is well-defined in either the strong or weak topology. We shall indicate the functional correspondence (7) of functions $f\left(e^{i \theta}\right)$ in the class $B(d E)$ to operators $F$ on $\mathscr{C}$ by the notation $f \sim F$.

2.2. We define the class $\mathscr{E}_{1}$ of bounded linear operators on $\mathscr{H}$ as follows:

$\left(\alpha_{1}\right) E_{\theta}(0 \leqq \theta \leqq 2 \pi)$ belongs to the class $\mathscr{E}_{1}=\mathscr{E}_{1}(\mathscr{L})$ whenever

(a) $E_{\theta}=E_{\theta}^{*}, 0 \leqq \theta \leqq 2 \pi$.

(b) $E_{\theta_{1}} E_{\theta_{2}}{ }^{-}=E_{\min \left(\theta_{1}, \theta_{2}\right)}, \theta_{1}, \theta_{2} \in[0,2 \pi]$.

(c) $E_{0+0}=E_{0}, 0 \leqq \theta<2 \pi$.

(d) $E_{0}=0$.

Note that (a) and (b) imply $E_{2 \pi} \leqq I$. If we also require $E_{2 \pi}=I$, then we obtain a subclass $\mathscr{E}_{0}$ of $\mathscr{E}_{1}$ which is the usual orthogonal spectral family or resolution of the identity for a unitary operator given by the spectral theorem (cf. [10, p. 281] or [20, p. 357]). Likewise, we note that $(\alpha \alpha)$ implies $E_{2 \pi} \leqq I$ for $E_{\theta} \in \mathscr{E}$, and if $E_{2 \pi}=I$, then $\mathscr{E}$ is the class of functions called a generalized spectral family [16, p. 6] or a generalized resolution of the identity [2, p. 121].

A theorem due to Naimark [16, p. 6] asserts that every generalized spectral family, can be represented as the projection of an orthogonal spectral family. Naimark's theorem has been extended to nonnormalized cases by McKelvey [8] and Berberian [3, Th. 1]. For our classes $\mathscr{E}$ and $\mathscr{E}_{1}$ it may be stated:

Theorem 1. (Naimark). Let $E_{\theta} \in \mathscr{E}(\mathscr{C})$. There exists a Hilbert 
space $\mathscr{H}^{+} \supseteqq \mathscr{H}$ and a projector valued $E_{\theta}^{+} \in \mathscr{E}_{1}\left(\mathscr{C}^{+}\right)$such that

(i) $E_{\theta} x=P E_{\theta}^{+} x$, for all $x \in \mathscr{C}$, where $P$ is the orthogonal projector onto the subspace $\mathscr{H}$ of $\mathscr{H}^{+}$.

(ii) $\mathscr{C}^{+}$is spanned by $\mathscr{\mathscr { C }} \cup\left\{E_{\theta}^{+} x: x \in \mathscr{C}, 0 \leqq \theta \leqq 2 \pi\right\}$, and

(iii) $E^{+}(\sigma)=0$ if and only if $E(\sigma)=0$, where $\sigma$ is any Borel set on $[0,2 \pi]$ and $E(\sigma)$ is the operator measure related to $E_{\theta}$. Also, $E_{2 \pi}^{+}=I$ if and only if $E_{2 \pi}=I$.

If $E_{\theta}^{+} \in \mathscr{E}_{1}$ is the minimal dilation of $E_{\theta} \in \mathscr{E}$, then (iii) of the Naimark dilation theorem implies that $\Lambda\left(E^{+}\right)=\Lambda(E)$, and hence

$$
B\left(d E^{+}\right)=B(d E) \text {. }
$$

For $f \sim F$ we define $F^{+}$by

$$
F^{+}=\int_{0}^{2 \pi} f\left(e^{i \theta}\right) d E_{\theta}^{+} .
$$

The Naimark theorem and equation (7) then give

$$
F x=P \int_{0}^{2 \pi} f\left(e^{i \theta}\right) d E_{\theta}^{+} x=P F^{+} x,
$$

for $x \in \mathscr{H}$, and

$$
(F x, y)=\left(F^{+} x, y\right)
$$

for $x, y \in \mathscr{Y}$.

2.3. The functional correspondence (7) has the following properties (cf. [8]): If $f \sim F, g \sim G, f_{n} \sim F_{n}$, and $g_{n} \sim G_{n}$, then

( i ) Linear: $c_{1} f+c_{2} g \sim c_{1} F+c_{2} G$, where $c_{1}, c_{2}$ are scalar constants.

(ii ) Preserves conjugates: $\bar{f} \sim F^{*}$.

(iii ) Positive: $f\left(e^{i \theta}\right) \geqq 0$ on $\Lambda(E)$ implies $F \geqq 0$.

(iv) When $E_{\theta}$ is projector-valued $\left(E_{\theta}=E_{\theta}^{*}=E_{\theta}^{2}\right)$, then the correspondence is multicative: $f g \sim F G$.

$$
\text { ( v ) } \quad\|F x\|^{2} \leqq \int_{0}^{2 \pi}\left|f\left(e^{i s}\right)\right|^{2} d\left(E_{\theta} x, x\right),
$$

for $x \in \mathscr{K}$, with equality when $E_{0}$ is projector-valued.

( vi ) Norm-decreasing: $\|F\| \leqq \operatorname{ess} \sup \left|f\left(e^{i \theta}\right)\right|$ on $\Lambda(E)$.

(vii) Strong convergence: When $f_{n}\left(e^{i \theta}\right) \rightarrow f\left(e^{i \theta}\right)$ boundedly a.e. $(d E)$, i.e., when $\left|f_{n}\left(e^{i \theta}\right)\right| \leqq M$ and $f_{n}\left(e^{i \theta}\right) \rightarrow f\left(e^{i \theta}\right)$ as $n \rightarrow \infty$ a.e. $(d E)$, then $F_{n} \rightarrow F$ strongly, i.e., $\left\|F_{n} x-F x\right\| \rightarrow 0$ for all $x \in \mathscr{C}$.

(viii) Uniform convergence: When $f_{n}\left(e^{i \theta}\right) \rightarrow f\left(e^{i \theta}\right)$ uniformly on $\Lambda(E)$ as $n \rightarrow \infty$, then $F_{n} \rightarrow F$ uniformly, i.e., $\left\|F_{n}-F\right\| \rightarrow 0$.

(ix) $1 \sim E_{2 \pi}$. 
2.4. Suppose that an $E_{\theta} \in \mathscr{E}$ is given. Define $R_{z}$ and $T^{(k)}$ by means of formulas (1) and (2), i.e., in terms of the functional correspondence (7)

$$
\frac{1}{1-e^{i \theta} z} \sim R_{z} \quad \text { and } \quad e^{i k \theta} \sim T^{(k)} .
$$

LEMmA 1. The operators $R_{z}$ and $T^{(k)}$ defined above belong to the classes $\mathscr{R}$ and $\mathscr{J}$ respectively. Furthermore, the functions $E_{\theta}, R_{z}$, and $T^{(k)}$ are interrelated by formulas (1)-(6).

Proof. ( i ) To show $R_{z} \in \mathscr{R}$ we verify conditions (a)-(c) of $(\beta)$.

(a) Since the integrand in formula (1) is holomorphic in $z$ and continuous in $\theta$ on a bounded contour, $R_{z} x$ is holomorphic for $|z| \neq 1$, $x \in \mathscr{H}$. Then $R_{z}$ is holomorphic for $|z| \neq 1$ (cf. [20, p. 206]).

(b) Note that

$$
\frac{1}{1-e^{i \theta} z}=\frac{1}{2}+\frac{1}{2} \frac{1+e^{i \theta} z}{1-e^{i \theta} z}=\frac{1}{2}+\frac{1}{2} \frac{1+2 i \operatorname{Im} e^{i \theta} z-|z|^{2}}{\left|1-e^{i \theta} z\right|^{2}},
$$

so

$$
\operatorname{Re} \frac{1}{1-e^{i \theta} z}=\frac{1}{2}+\frac{1}{2} \frac{1-|z|^{2}}{\left|1-e^{i \theta} z\right|^{2}} .
$$

Integrating this identity with respect to $d\left(E_{\theta} x, x\right), 0 \leqq \theta \leqq 2 \pi$, and using the linearity, one obtains

$$
\operatorname{Re}\left(R_{z} x, x\right)=\frac{1}{2} \int_{0}^{2 \pi} d\left(E_{0} x, x\right)+\frac{1}{2}\left(1-|z|^{2}\right) \int_{0}^{2 \pi} \frac{1}{\left|1-e^{i \theta} z\right|^{2}} d\left(E_{\theta} x, x\right) .
$$

Now the first integral on the right is $\left(R_{0} x, x\right)$ by formula (1) for $z=0$. Then

$$
\frac{2 \operatorname{Re}\left(R_{z} x, x\right)-\left(R_{0} x, x\right)}{1-|z|^{2}}=\int_{0}^{2 \pi} \frac{1}{\left|1-e^{i \theta} z\right|^{2}} d\left(E_{\theta} x, x\right) \geqq\left\|R_{z} x\right\|^{2},
$$

for $|z| \neq 1, x \in \mathscr{K}$, by property (v) of $\S 2.3$.

(c) Suppose that $z \neq 0,|z| \neq 1$, and $x, y \in \mathscr{K}$. By equation (1)

$$
\left(R_{z^{*}} x, y\right)=\int_{0}^{2 \pi} \frac{1}{1-e^{i \theta} z^{*}} d\left(E_{\theta} x, y\right),
$$

where $z^{*}=1 / \bar{z}$. Then using properties (i) and (ii) of $\S 2.3$

$$
\begin{aligned}
\left(R_{z}^{*} x, y\right) & =\int_{0}^{2 \pi} \overline{\left[\frac{1}{1-e^{i \theta} z}\right]} d\left(E_{\theta} x, y\right)=\int_{0}^{2 \pi} \frac{1}{1-e^{-\imath \theta} \bar{z}} d\left(E_{\theta} x, y\right) \\
& =\int_{0}^{2 \pi} d\left(E_{\theta} x, y\right)-\int_{0}^{2 \pi} \frac{1}{1-e^{i \theta} z^{*}} d\left(E_{\theta} x, y\right) \\
& =\left(R_{0} x, y\right)-\left(R_{z^{*}} x, y\right)=\left(\left[R_{0}-R_{z^{*}}\right] x, y\right),
\end{aligned}
$$


i.e., $R_{z^{*}}=R_{0}-R_{z}^{*}$.

(ii) $T^{(k)} \in \mathscr{T}$ :

By (2) for $k=0$ and $(i x)$ of $\S 2.3$ we have $T^{(0)}=E_{2 \pi} \leqq I$. That formula (2) satisfies $(\gamma b)$ is not new (cf. [3, Th. 4]).

(iii) Related by formulas (1)-(6):

(a) Since the function $1 /\left(1-e^{i \theta} z\right)$ is holomorphic for $|z| \neq 1$, it has Laurent series expansions for $|z|<1$ and $|z|>1$. Using properties (i) and (viii) of $\S 2.3$ and formulas (1) and (2) on these Laurent expansions, we obtain formula (3). Clearly, formula (6) is an inversion of formula (3).

(b) To prove (4), let $0<\varphi \leqq 2 \pi, 0 \leqq r<1$, and let $\theta_{1}, \theta_{2}$ be points of continuity of $E_{\theta}$. Define

$$
\begin{aligned}
f\left(r, e^{i \varphi}\right) & \equiv \frac{1}{\pi} \int_{\theta_{1}}^{\theta_{2}} \operatorname{Re} \frac{1}{1-e^{i \varphi}\left(r e^{-i \theta}\right)} d \theta-\frac{\left(\theta_{2}-\theta_{1}\right)}{2 \pi} \\
& =\frac{1}{2 \pi} \int_{\theta_{1}}^{\theta_{2}} \operatorname{Re}\left\{\frac{2}{1-r e^{i(\varphi-\theta)}}-1\right\} d \theta \\
& =\frac{1}{2 \pi} \int_{\theta_{1}}^{\theta_{2}} \operatorname{Re} \frac{1+r e^{i(\varphi-\theta)}}{1-r e^{i(\varphi-\theta)}} d \theta=\frac{1}{2 \pi} \int_{\theta_{1}}^{\theta_{2}} P_{r}(\varphi-\theta) d \theta,
\end{aligned}
$$

where $P_{r}$ is Poisson's kernel $\left[7\right.$, p. 30]. Then $\left|f\left(r, e^{i \varphi}\right)\right| \leqq 1$, and by Fatou's theorem (cf. [7, p. 34])

$$
\begin{aligned}
\operatorname{Lim}_{r-1^{-}} f\left(r, e^{i \varphi}\right) & =1 \text { if } \varphi \in\left(\theta_{1}, \theta_{2}\right) \\
& =0 \text { if } \varphi \notin\left[\theta_{1}, \theta_{2}\right],
\end{aligned}
$$

i.e., $f$ approaches $\chi(\Delta ; \varphi)$ a.e., $0<\varphi \leqq 2 \pi$, where $\chi(\Delta ; \varphi)$ is the characteristic function of the interval $\Delta=\left(\theta_{1}, \theta_{2}\right]$.

Define

$$
F(r) \equiv \int_{0}^{2 \pi} f\left(r, e^{i \varphi}\right) d E_{\varphi} .
$$

Then by property (vii) of $\S 2.3$

$$
\operatorname{Lim}_{r \rightarrow 1^{-}} F(r)=\int_{0}^{2 \pi} \chi(\Delta ; \phi) d E_{\varphi}=E_{\theta_{2}}-E_{\theta_{1}} .
$$

But for $0 \leqq r<1$

$$
\begin{aligned}
F(r) & =\int_{0}^{2 \pi} \frac{1}{2 \pi} \int_{\theta_{1}}^{\theta_{2}} \operatorname{Re}\left\{\frac{2}{1-r e^{i(\varphi-\theta)}}-1\right\} d \theta d E_{\varphi} \\
& =\frac{1}{2 \pi} \int_{\theta_{1}}^{\theta_{2}} \int_{0}^{2 \pi} \operatorname{Re}\left\{\frac{2}{1-r e^{-i \theta} e^{i \varphi}}-1\right\} d E_{\varphi} d \theta \\
& =\frac{1}{2 \pi} \int_{\theta_{1}}^{\theta_{2}}\left(2 \operatorname{Re} R_{r \exp (-i \theta)}-R_{0}\right) d \theta
\end{aligned}
$$


which implies (4). The interchanging of the order of integration is justified because the integrand is continuous with respect to $\theta, \varphi$.

(c) Similarly, (5) may be seen to be an inversion of (2) by showing

$$
\begin{aligned}
\frac{1}{2 \pi} \sum_{k=-\infty}^{\infty} \frac{e^{-i k \theta_{1}}-e^{-i k \theta_{2}}}{i k} T^{\langle k)} & =\frac{1}{2 \pi} \operatorname{Lim}_{r-1^{-}} \int_{0}^{2 \pi} \int_{\theta_{1}}^{\theta_{2}} P_{r}(\varphi-\theta) d \theta d E_{\varphi} \\
& =E_{\theta_{2}}-E_{\theta_{1}} .
\end{aligned}
$$

2.5 Theorem A. Each function $E_{\theta} \in \mathscr{E}, R_{z} \in \mathscr{R}$, or $T^{(k)} \in \mathscr{T}$ belongs to a unique triple of functions $\left\{E_{\theta}, R_{z}, T^{(k)}\right\}$ in $\mathscr{S} \equiv$ $\{\mathscr{E}, \mathscr{R}, \mathscr{T}\}$, such that the members of the triple are interrelated by formulas (1)-(6).

Proof. Due to Lemma 1 we need only show that each $R_{z} \in \mathscr{R}$ can be expressed by equation (1), and each $T^{(k)} \in \mathscr{T}$ by equation (2), in terms of some unique $E_{\theta} \in \mathscr{E}$. This is done in (i) and (ii) below.

(i) Suppose $R_{z} \in \mathscr{R}$, i.e., $R_{z}$ satisfies the conditions $(\beta)$. Then $R_{z}$ satisfies the following "weak" properties:

$\left(\beta^{*}\right)$ (a) $\left(R_{z} x, x\right)$ is a holomorphic function of $z$ for $|z| \neq 1, x \in \mathscr{K}$.

(b) $\operatorname{Re}\left(R_{z} x, x\right) \lesseqgtr \frac{1}{2}\left(R_{0} x, x\right),|z| \gtrless 1, x \in \mathscr{H}$.

(c) $\left(R_{0} x, x\right) \leqq(x, x), x \in \mathscr{H}$.

(d) $\quad\left(\left[R_{0}-R_{z^{*}}\right] x, x\right)=\overline{\left(R_{z} x, x\right)}, z \neq 0,|z| \neq 1, x \in \mathscr{X}$.

(a), (b), and (d) are immediate from $(\beta)$ and the fact that

$$
\frac{1}{2}\left(1-|z|^{2}\right)\left\|R_{z} x\right\|^{2} \leqq 0 \text { for }|z| \gtrless 1, x \in \mathscr{C} \text {. }
$$

By $(\beta b)$ with $z=0$

$$
\left\|R_{j} x\right\|^{2} \leqq\left(R_{0} x, x\right) \leqq\left|\left(R_{0} x, x\right)\right| \leqq\left\|R_{0} x\right\|\|x\| .
$$

Hence $\left\|R_{x} x\right\| \leqq\|x\|$, and $\left(R_{0} x, x\right) \leqq\|x\|^{2}$.

Define

$$
\varphi(z) \equiv-\left(R_{0} x, x\right)+2\left(R_{z} x, x\right), \text { for }|z| \neq 1 .
$$

Since $\left(R_{z} x, x\right)$ is holomorphic in $|z| \neq 1$, and $\left(R_{0} x, x\right)$ is a constant with respect to $z$, it is clear that $\varphi(z)$ is holomorphic in $|z| \neq 1$. Now

$$
\operatorname{Re} \varphi(z)=-\left(R_{0} x, x\right)+2 \operatorname{Re}\left(R_{z} x, x\right),
$$

so by $\left(\beta^{*} \mathrm{~b}\right), \operatorname{Re} \varphi(z) \geqq 0$ for $|z|<1$, and $\operatorname{Re} \varphi(z) \leqq 0$ for $|z|>1$. Also, using $\left(\beta^{*} c\right), \varphi(0)=\left(R_{0} x, x\right) \leqq(x, x)$; and by $\left(\beta^{*} \mathrm{~d}\right)$

$$
\varphi\left(z^{*}\right)=-\left(R_{0} x, x\right)+2\left(R_{z^{*}} x, x\right)=\left(\mathrm{R}_{0} x, x\right)-2 \overline{\left(R_{z} x, x\right)}=-\overline{\varphi(z)},
$$

for $z \neq 0$ and $|z| \neq 1$. Thus if $R_{z} \in \mathscr{R}$, then $\varphi(z)$ satisfies

( $\left.\beta^{\prime}\right)$ (a) $\varphi(z)$ is holomorphic in $|z| \neq 1$. 
(b) $\operatorname{Re} \varphi(z) \lesseqgtr 0$ for $|z| \gtrless 1$.

( c ) $\varphi(0) \leqq(x, x)$ (i.e., $\varphi(0)$ is real and $\varphi(0) \leqq(x, x)$ ).

(d) $\varphi\left(z^{*}\right)=-\overline{\varphi(z),} z \neq 0,|z| \neq 1$.

Now the conditions $\left(\beta^{\prime}\right)$ are sufficient to apply a theorem due to Herglotz [5] (see also [9, pp. 58-60], or [2, p. 5] with $t$ replaced by $2 \pi-\theta$ ) which says that $\varphi(z)$ is of the form

$$
\varphi(z)=\int_{0}^{2 \pi} \frac{1+e^{i \theta} z}{1-e^{i \theta} z} d \sigma(\theta)+i \operatorname{Im} \varphi(0)
$$

$|z|<1$, where $\sigma(\theta), 0 \leqq \theta \leqq 2 \pi$, is a real nondecreasing function of bounded variation, and $\operatorname{Im} \varphi(0)=0$, since $\varphi(0)$ is real.

If $|z|<1$, then by $(10)$ and $\left(\beta^{\prime} d\right)$

$$
\varphi\left(z^{*}\right)=-\overline{\varphi(z)}=-\int_{0}^{2 \pi} \frac{1+e^{-i \theta} \bar{z}}{1-e^{-i 0} \bar{z}} d \sigma(\theta)=\int_{0}^{2 \pi} \frac{1+e^{i \theta} z^{*}}{1-e^{i \theta} z^{*}} d \sigma(\theta) .
$$

If $|z|<1$, then $\left|z^{*}\right|>1$. Therefore, formula (10) is valid for all $|z| \neq 1$.

From the definition of $\varphi(z)$

$$
\left(R_{z} x, x\right)=\frac{1}{2}\left(R_{0} x, x\right)+\frac{1}{2} \varphi(z)=\frac{1}{2} \varphi(0)+\frac{1}{2} \varphi(z), \quad|z| \neq 1,
$$

so

$$
\left(R_{z} x, x\right)=\frac{1}{2} \int_{0}^{2 \pi} d \sigma(\theta)+\frac{1}{2} \int_{0}^{2 \pi} \frac{1+e^{i \theta} z}{1-e^{i \theta} z} d \sigma(\theta)=\int_{0}^{2 \pi} \frac{1}{1-e^{i \theta} z} d \sigma(\theta),
$$

for $|z| \neq 1, x \in \mathscr{C}$.

Under the normalization conditions

$$
\begin{aligned}
& \sigma(0)=0 \\
& \sigma(\theta)=\sigma(\theta+0), 0 \leqq \theta<2 \pi,
\end{aligned}
$$

where $\sigma(\theta+0) \equiv \operatorname{Lim} \sigma(\varphi)$ as $\varphi \rightarrow \theta^{+}$, the real nondecreasing function $\sigma(\theta)=\sigma(\theta ; x)$ in (10) is uniquely determined by $\varphi(z)$ or $\left(R_{z} x, x\right)$ (cf. [2, pp. 3-7]).

Defining the function $\sigma(\theta ; x, y)$ for $x, y \in \mathscr{C}$ by the polarization formula

$$
\begin{aligned}
\sigma(\theta ; x, y) \equiv & \frac{1}{4}[\sigma(\theta ; x+y)-\sigma(\theta ; x-y) \\
& +i \sigma(\theta ; x+i y)-i \sigma(\theta ; x-i y)],
\end{aligned}
$$

and using the analogous formula for the bilinear form $\left(R_{z} x, y\right)$, one obtains

$$
\left(R_{z} x, y\right)=\int_{0}^{2 \pi} \frac{1}{1-e^{i \theta} z} d \sigma(\theta ; x, y), \quad|z| \neq 1, x, y \in \mathscr{L} .
$$


The complex-valued functions $\sigma(\theta ; x, y)$ also satisfy the normalization conditions (11) and are uniquely determined by equation (12). In particular, one has $\sigma(\theta ; x, x)=\sigma(\theta ; x)$. It follows by an elementary argument (cf. $[2, \S 65])$ that $\sigma(\theta ; x, y)$ is a symmetric bilinear functional of $x, y$ and

$$
|\sigma(\theta ; x, x)| \leqq \sigma(2 \pi ; x, x)=\int_{0}^{2 \pi} d \sigma(\theta ; x, x)=\left(R_{0} x, x\right) \leqq(x, x) .
$$

By the theorem on the general form of a symmetric bilinear functional [1, §21], there exists a uniquely determined family of bounded self-adjoint linear operators $E_{\theta}$ which depend on the parameter $\theta(0 \leqq \theta \leqq 2 \pi)$ such that $\sigma(\theta ; x, y)=\left(E_{\theta} x, y\right)$ for all $x, y \in \mathscr{L}$. It remains to show $E_{\theta} \in \mathscr{E}$, i.e., $E_{\theta}$ satisfies conditions (a)-(c) of $(\alpha)$. But since $\left(E_{\theta} x, x\right)=\sigma(\theta ; x, x)=\sigma(\theta)$, this follows from the normalization conditions (11) and a simple argument showing that if $\left(E_{\theta} x, x\right)$ is weakly continuous from the right, then it is strongly continuous from the right.

Equation (12) becomes equation (1) in the weak sense, hence in the strong sense, since both interpretations of the integral make sense and define the same operator $R_{z}$. Then $E_{\theta}$ is completely defined by the operator $R_{z}$, and in turn $E_{\theta}$ completely defines $R_{z}$ by (1). This proves (i).

Since the construction of $E_{\theta}$ only depends upon the "weak" properties $\left(\beta^{*}\right)$, we obtain as a byproduct of the proof the result:

THEOREM 2. The class $\mathscr{R}$ may be characterized by the properties of $\left(\beta^{*}\right)$ as well as those of $(\beta)$.

(ii) Suppose $T^{(k)} \in \mathscr{T}$, i.e., $T^{(k)}$ satisfies the conditions $(\gamma)$. Then by a generalization of Naimarks moment theorem [3, Ths. 3 and 4], or by an argument similar to $[2, \S 62]$ we obtain

$$
T^{(k)}=\int_{0}^{2-} e^{i k \theta} d E_{\theta}^{\prime} \quad( \pm k=0,1,2, \cdots),
$$

where $E_{\theta}^{\prime} \in \mathscr{E}$.

It remains to show $E_{\theta} \equiv E_{\theta}^{\prime}$. But this follows from formulas (1)(6). For example, by substituting (1) and (2) into (3) and using the normalization conditions. This proves Theorem A.

3. The generalized resolvent.

3.1 Remarks. If $\left\{E_{\theta}, R_{z}, T^{(k)}\right\}$ is a matched triple of functions in $\mathscr{S}$, then

(i) $R_{0}=T^{(0)}=E_{2 \pi} \leqq I$.

(ii) $T^{-k)}=\left[T^{(k)}\right]^{*}, \pm k=0,1,2, \cdots$.

(iii) $\left|\left(T^{(k)} x, x\right)\right| \leqq\left(T^{(0)} x, x\right), x \in \mathscr{C}, \pm k=0,1,2, \cdots$. 


$$
\left\|T^{(k)}\right\| \leqq\left\|T^{(0)}\right\| \leqq 1, \pm k=0,1,2, \cdots
$$

Proof. (i) and (iii) follow from equations (1) and (2). (ii) follows by the functional calculus from $e^{-i k \theta}=\overline{e^{i k v}}$. (iv) is proved in [3, corollary to Th. 2].

3.2 THeOREM 3. Let $\left\{E_{\theta}, R_{z}, T^{(k)}\right\}$ be a matched triple of functions in $\mathscr{S}$, and let $N=N\left(E_{2 \pi}\right)$ be the null-space of $E_{2 \pi}$. Then

( i ) $N\left(E_{\theta_{1}}\right) \supseteqq N\left(E_{\theta_{2}}\right) \supseteqq N$ and $\overline{E_{\theta_{1}} \mathscr{H}} \cong \overline{E_{\theta_{2}} \mathscr{C}} \cong \overline{E_{2 \pi} \mathscr{C}}=N^{\mathrm{i}}$, for $0 \leqq \theta_{1}<\theta_{2} \leqq 2 \pi$.

(ii) $N\left(T^{(k)}\right) \supseteqq N\left(T^{(0)}\right)=N$ and $\overline{T^{(k)} \mathscr{\mathscr { C }}} \cong \overline{T^{(0)} \mathscr{\mathscr { C }}}=N^{\perp}, \quad \pm k=$ $0,1 \cdots$.

(iii) $N\left(R_{z^{*}}\right) \supseteqq N\left(R_{z}\right)=\left\{x:\left(R_{z} x, x\right)=0\right\}=N$ and $\overline{R_{z^{*}} \mathscr{\mathscr { C }}} \leqq \overline{R_{z} \mathscr{\mathscr { C }}}=$ $N^{\perp}$, for $|z|<1$.

In particular, the decomposition $\mathscr{H}=N^{\perp}+N$ is reducing for all values of the functions $E_{\theta}, R_{z}, T^{(k)}$, and these functions vanish identically on $N$.

Proof. (i) Since $E_{0}$ is nonnegative, the Cauchy-Schwartz inequality,

$$
\left|\left(E_{\theta} x, y\right)\right|^{2} \leqq\left(E_{\theta} x, x\right)\left(E_{\theta} y, y\right),
$$

is valid and shows that $N\left(E_{\theta}\right)=\left\{x:\left(E_{\theta} x, x\right)=0\right\}$. Also, $\overline{E_{\theta} \mathscr{C}}=$ $N\left(E_{\theta}\right)^{\perp}$. The assertions then follow from $(\alpha \mathrm{a})$.

(ii) If $T^{(0)} x=0$, then $E_{2 \pi} x=0$ by remark (i) of $\S 3.1$. Hence $E_{\theta} x \equiv 0,0 \leqq \theta \leqq 2 \pi$, by (i) above. Then equation (2) gives $T^{(k)}=0$, $\pm k=0,1,2, \cdots$. Also,

$$
\overline{T^{(k)} \mathscr{\mathscr { C }}}=N\left(T^{(k) *}\right)^{\perp}=N\left(T^{(-k)}\right)^{\perp} \subseteq N\left(T^{(0)}\right)^{\perp}=\overline{T^{(0)} \mathscr{\mathscr { C }}} .
$$

(iii) Suppose $|z|<1$. By $(\beta \mathrm{b}) \operatorname{Re}\left(R_{z} x, x\right) \geqq \frac{1}{2}\left(R_{0} x, x\right)$. Therefore, if $\left(R_{z_{0}} x, x\right)=0$ for some $z_{0},\left|z_{0}\right|<1$, then $\operatorname{Re}\left(R_{z_{0}} x, x\right)=0$, and $\left(R_{0} x, x\right)=$ $\left(E_{2 \pi} x, x\right)=0$. Thus $E_{2 \pi} x=0$, and $E_{0} x \equiv 0,0 \leqq \theta \leqq 2 \pi$, by (i) above. But then equation (1) gives $R_{z} x=0,|z|<1$. Clearly $R_{z} x=0$ implies $\left(R_{z} x, x\right)=0$. It follows that $N\left(R_{z}\right)=\left\{x:\left(R_{z} x, x\right)=0\right\}=N,|z|<1$.

Since $\operatorname{Re}\left(R_{z}^{*} x, x\right)=\operatorname{Re}\left(R_{z} x, x\right)$, the above argument shows that if $\left(R_{z_{0}}^{*} x, x\right)=0$ for some $z_{0},\left|z_{0}\right|<1$, then $E_{0} x \equiv 0,0 \leqq \theta \leqq 2 \pi$. But then the adjoint of formula (1) gives $R_{z}^{*} x=0,|z|<1$. Hence $N\left(R_{z}^{*}\right)=$ $N,|z|<1$.

By $(\beta \mathrm{c}) R_{z} x=0,|z|<1$, implies $R_{z^{*}} x=0,|z|<1$, i.e., $N\left(R_{z^{*}}\right) \supseteqq$ $N\left(R_{z}\right)|z|<1$. It is possible for $N\left(R_{z^{*}} \supset N\left(R_{z}\right),|z|<1\right.$. For example,

$$
\begin{array}{rlrl}
R_{z} & \equiv R_{0}, & & |z|<1 \\
& \equiv 0, & |z|>1
\end{array}
$$


belongs to the class $\mathscr{R}$, and $N\left(R_{z^{*}}\right)=\mathscr{C} \supset N\left(R_{z}\right),|z|<1$, if $R_{0} \neq 0$. Finally, for $|z|<1$

$$
\overline{R_{z^{*}} \mathscr{\mathscr { C }}}=N\left(R_{z^{*}}^{*}\right)^{\perp}=N\left(R_{0}-R_{z}\right)^{\perp} \leqq N\left(R_{z}\right)^{\perp}=N\left(R_{z}^{*}\right)^{\perp}=\overline{R_{z} \mathscr{\mathscr { C }}}=N^{\perp} \text {. }
$$

By (i)-(iii) $N^{\perp}$ and $N$ and invariant subspaces for $E_{\theta}, R_{z}, T^{(k)}$, and, consequently, the decomposition is reducing.

Definition. A matched triple of functions $\left\{E_{\theta}, R_{z}, T^{(k)}\right\}$ is said to be proper whenever $N=\{0\}$.

Remarks. 1. Any matched triple generates a proper triple on the reducing subspace.

2. For $E_{\theta} \in \mathscr{E}_{1}$, the corresponding matched triple is proper if and only if $E_{2 \pi}=I$.

3.3 Definition. The linear operator $T$ of the Hilbert space $\mathscr{C}$ is said to be a contraction operator if $\|T x\| \leqq\|x\|$ for all $x \in D_{T}$, where $D_{T}=$ domain of $T=\mathscr{C}$, i.e., $\|T\| \leqq 1$.

Definition. The linear operator $T$ is said to be an isometric operator if $(T x, T y)=(x, y)$ for all $x, y \in D_{T}$. If, in addition, $D_{T}=\Delta_{T} \equiv$ range of $T=\mathscr{H}$, then $T$ is said to be unitary.

Suppose $T$ is a contraction operator on the Hilbert space $\mathscr{H}$. Then the resolvent operator

$$
r(z) \equiv(I-z T)^{-1}, \quad|z|<1,
$$

exists as a bounded operator with domain $J_{T}(z) \equiv$ range $(I-z T)=$ $(I-z T) D_{T}=\mathscr{H}$.

Lemma 2. (a) If $\|T\| \leqq 1, D_{T}=\mathscr{H}$, then $T$ has resolvent $r(z)$ which satisfies

$$
\operatorname{Re}(r(z) x, x) \geqq \frac{1}{2}\|x\|^{2}+\frac{1}{2}\left(1-|z|^{2}\right)\|r(z) x\|^{2},
$$

$|z|<1, x \in \mathscr{X}$, or the equivalent

$$
\|[r(z)-I] x\| \leqq|z|\|r(z) x\|, \quad|z|<1, x \in \mathscr{\mathscr { C }} .
$$

Furthermore, r(z) satisfies

$$
(1-|z|)|| r(z)-I \| \leqq|z|, \quad|z|<1 .
$$

(b) If $T$ has resolvent $r(z)$ which satisfies equation (13) (or $\left(13^{\prime}\right)$ ) for a single $z_{0}$ in $0<\left|z_{0}\right|<1$ and $x \in \mathscr{C}$, then $\|T\| \leqq 1$, 
$D_{T}=\mathscr{X}$.

Proof. (a) For $y \in D_{T}=\mathscr{C}$

$$
\|[I-z T] y\|^{2}=2 \operatorname{Re}(y,[I-z T] y)+|z|^{2}\|T y\|^{2}-\|y\|^{2} .
$$

If $T$ is a contraction operator, then

$$
\|[I-z T] y\|^{2} \leqq 2 \operatorname{Re}(y,[I-z T) y)+\left(|z|^{2}-1\right)\|y\|^{2}
$$

for $|z|<1$. Letting $x=[I-z T] y, y=r(z) x$ in this equation, we obtain (13). Now

$$
\|r(z) x-I x\|^{2}=\|r(z) x\|^{2}-2 \operatorname{Re}(r(z) x, x)+\|x\|^{2},
$$

so $(13)$ is equivalent to $\left(13^{\prime}\right)$.

Nothing that $\|T\| \leqq 1, D_{T}=\mathscr{C}$, implies

$$
\|[I-z T] y\| \geqq(1-|z|)\|y\|,
$$

and letting $x=[I-z T] y, y=r(z) x$ in this inequality, we obtain $(1-|z|)\|r(z) x\| \leqq\|x\|$ for all $x \in \mathscr{K}=\Delta_{T}(z)$, i.e.,

$$
(1-|z|)\|r(z)\| \leqq 1 \text {. }
$$

Using (16) in $\left(13^{\prime}\right)$, we have (14).

(b) Suppose an operator $T$ has resolvent $r(z)$ which satisfies $\left(13^{\prime}\right)$ (or (13)) for some $z_{0}$ in $0<\left|z_{0}\right|<1$. Now

$$
\left\|r\left(z_{0}\right) x-I x\right\|=\left\|r\left(z_{0}\right) x-\left(I-z_{0} T\right) r\left(z_{0}\right) x\right\|=\left|z_{0}\right|\left\|\operatorname{Tr}\left(z_{0}\right) x\right\|,
$$

so $\left(13^{\prime}\right)$ gives

$$
\left|z_{0}\right|\left\|\operatorname{Tr}\left(z_{0}\right) x\right\| \leqq\left|z_{0}\right|\left\|r\left(z_{0}\right) x\right\|
$$

for $0<\left|z_{0}\right|<1, x \in \mathscr{K}$. Hence $\|T\| \leqq 1$.

3.4 Theorem B. $R_{z} \in \mathscr{R}$ and $R_{0}=I$ if and only if

$$
\begin{aligned}
R_{z} & =\left(I-z T_{z}\right)^{-1}, \quad|z|<1 \\
& =I-\left(I-z^{-1} T_{z^{*}}^{*}\right)^{-1}, \quad|z|>1,
\end{aligned}
$$

where

(i ) ||$T_{z} \| \leqq 1,|z|<1, D\left(T_{z}\right)=\mathscr{C}$

(ii) $T_{z}$ is a holomorphic function of $z$ for $|z|<1$.

Proof. Suppose

$$
\begin{aligned}
R_{z} & =\left(I-z T_{z}\right)^{-1}, \quad|z|<1 \\
& =I-\left(I-z^{-1} T_{z^{*}}^{*}\right)^{-1}, \quad|z|>1,
\end{aligned}
$$


where $T_{z}$ satisfies (i) and (ii). Then clearly, $D\left(R_{z}\right)=\mathscr{H}$, and $R_{0}=$ $I^{-1}=I$.

(a) By (i)

$$
\begin{aligned}
\left\|\left[I-z T_{z}\right] x\right\| & \geqq\|x\|-|z|\left\|T_{z} x\right\| \\
& \geqq(1-|z|)\|x\|, \quad|z|<1,
\end{aligned}
$$

so $R_{z}$ not only exists but is bounded for $|z|<1$. Then $R_{z}=\left(I-z T_{z}\right)^{-1}$ is holomorphic for $|z|<1, x \in \mathscr{C}$, because $T_{z}$ is holomorphic by (ii).

Now $\left\|T_{z}^{*}\right\|=\left\|T_{z}\right\| \leqq 1,|z|<1, D\left(T_{z^{*}}\right)=\mathscr{H}$, so

$$
\begin{aligned}
\left\|\left[I-z^{-1} T_{z^{*}}^{*}\right] x\right\| & \geqq\|x\|-\left|z^{-1}\right|\left\|T_{z^{*}}^{*} x\right\| \\
& \geqq\left(1-\left|z^{-1}\right|\right)\|x\|, \quad|z|>1 .
\end{aligned}
$$

Thus $\left[\mathrm{I}-z^{-1} T_{z^{*}}^{*}\right]^{-1}$ exists and is bounded for $|z|>1$ with domain $\mathscr{H}$. Also, it follows from (ii) that $T_{z^{*}}^{*}$ is holomorphic for $|z|>1$. Then $R_{z}=I-\left(I-z^{-1} T_{z^{*}}^{*}\right)^{-1}$ is holomorphic for $|z|>1, x \in \mathscr{X}$. We have shown that $R_{z}$ is a holomorphic function of $z$ for $|z| \neq 1$.

(b) Since $R_{z}^{*}=\left[\left(I-z T_{z}\right)^{-1}\right]^{*}=\left(I-\bar{z} T_{z}^{*}\right)^{-1},|z|<1$, we have

$$
I-R_{z}^{*}=I-\left(I-\bar{z} T_{z}^{*}\right)^{-1}=R_{z^{*}}, \quad|z|<1, z \neq 0 .
$$

Similarily, for $|z|>1$

$$
R_{z}^{*}=\left[I-\left(I-z^{-1} T_{z^{*}}^{*-1}\right]^{*}=I-\left(I-z^{*} T_{z^{*}}\right)^{-1},\right.
$$

so

$$
I-R_{z}^{*}=\left(I-z^{*} T_{z^{*}}\right)^{-1}=R_{z^{*}}, \quad|z|>1 .
$$

We have proved that $R_{z^{*}}=I-R_{z}^{*},|z| \neq 1, z \neq 0$.

(c) By (i) and Lemma 2(a), $T_{z}$ has resolvent $R_{z}$ satisfying (13) and $\left(13^{\prime}\right)$ for all $|z|<1, \quad x \in \mathscr{C}$. Now $\left\|T_{z}^{*}\right\| \leqq 1,|z|<1$, and $D\left(T_{\bar{z}}^{*}\right)=\mathscr{C}$, so that Lemma 2(a) also applies to $T_{\bar{z}}^{*}$. Then $\left(I-z T_{\bar{z}}^{*}\right)^{-1}$ satisfies $\left(13^{\prime}\right)$ for $|z|<1$, i.e.,

$$
\left\|\left[\left(I-z T_{\bar{z}}^{*}\right)^{-1}-I\right] x\right\| \leqq|z|\left\|\left(I-z T_{\bar{z}}^{*}\right)^{-1} x\right\|, \quad|z|<1 .
$$

Replacing $z$ by $z^{-1}$ in this relation and nothing that $\left(I-z^{-1} T_{z^{*}}^{*}\right)^{-1}=R_{z^{*}}^{*}$, $|z|>1$, we obtain

$$
\left\|\left[R_{z^{*}}^{*}-I\right] x\right\| \leqq\left|z^{-1}\right|\left\|R_{z^{*}}^{*} x\right\|, \quad|z|>1 .
$$

Thus $R_{z^{*}}^{*}$ satisfies $\left(13^{\prime}\right)$ (and (13)) for all $|z|>1$. But $R_{z^{*}}^{*}=I-R_{z}$, $|\boldsymbol{z}|>1$, by (b) above. Hence $\left(13^{\prime}\right)$ becomes

$$
\left\|\left[\left(I-R_{z}\right)-I\right] x\right\| \leqq\left|z^{-1}\right|\left\|\left[I-R_{z}\right] x\right\|, \quad|z|>1,
$$

or

$$
\left\|\left[R_{z}-I\right] x\right\| \geqq|z|\left\|R_{z} x\right\|, \quad|z|>1,
$$


which is equivalent to

$$
\operatorname{Re}\left(R_{z} x, x\right) \leqq \frac{1}{2}\|x\|^{2}+\frac{1}{2}\left(1-|z|^{2}\right)\left\|R_{z} x\right\|^{2}, \quad|z|>1 .
$$

We have shown that $R_{z}$ satisfies $(\beta \mathrm{b})$ for all $|z| \neq 1$.

Combining (a)-(c) above, we have $R_{z} \in \mathscr{R}$ and $R_{0}=I$.

Conversely, suppose $R_{z} \in \mathscr{R}$ and $R_{0}=I$. By Theorem 3 (iii), $N\left(R_{z}\right)=N\left(R_{0}\right)=\{0\}$, so $R_{z}$ is proper, and $R_{z}^{-1}$ exists, $|z|<1$. In addition $N\left(R_{z}^{*}\right)=N\left(R_{z}\right)=\{0\}$, so $\left(R_{z}^{*}\right)^{-1}$ exists, $|z|<1$. By $(\beta \mathrm{b})$ for $|z|<1$ we have

$$
\frac{1}{2}\|x\|^{2} \leqq \operatorname{Re}\left(R_{z} x, x\right) \leqq\left|\left(R_{z} x, x\right)\right| \leqq\left\|R_{z} x\right\|\|x\|,
$$

so that

$$
\left\|R_{z}\right\| \geqq \frac{1}{2}\|x\|, \quad \text { for all } x \in \mathscr{C},|z|<1 .
$$

This implies that $R_{z}^{-1}$ exists and is bounded for $|z|<1$. Therefore, $\left(I-R_{z}^{-1}\right)=\left(R_{z}-I\right) R_{z}^{-1}$ is holomorphic in $z$ for $|z|<1$ because $R_{z}$ is. Also,

$$
\operatorname{Lim}_{z \rightarrow 0}\left(R_{z}-I\right) R_{z}^{-1}=0 .
$$

Then the function $z^{-1}\left(R_{z}-I\right) R_{z}^{-1}$ is holomorphic in $z$ for $|z|<1$, because the apparent singularity at $z=0$ is removable by making it continuous at $z=0$. Consequently, one may define $T_{z}$ to be the following function holomorphic for $|z|<1$ :

$$
\begin{aligned}
& T_{z} \equiv \frac{1}{z}\left(I-R_{z}^{-1}\right) \quad \text { for } \quad 0<|z|<1 \\
& T_{0} \equiv \operatorname{Lim}_{z \rightarrow 0} T_{z} .
\end{aligned}
$$

Then

$$
R_{z}=\left(I-z T_{z}\right)^{-1}, \quad|z|<1 .
$$

Since $\left(R_{z}^{*}\right)^{-1}$ exists we have

$$
T_{z}^{*}=\frac{1}{\bar{z}}\left[I-\left(R_{z}^{*}\right)^{-1}\right], \quad|z|<1 .
$$

Hence

$$
\begin{aligned}
R_{z}^{*} & =\left(I-\bar{z} T_{z}^{*}\right)^{-1}, \quad|z|<1 \\
& =I-R_{z^{*}}, \quad|z|<1
\end{aligned}
$$

by $(\beta c)$, and

$$
R_{z^{*}}=I-\left(I-\bar{z} T_{z}^{*}\right)^{-1}, \quad|z|<1,
$$

i.e., 


$$
R_{z}=I-\left(I-z^{-1} T_{z^{*}}^{*}\right)^{-1}, \quad|z|>1 .
$$

Since $(\beta \mathrm{b})$ with $R_{0}=I$ and $|z|<1$ is (13), we see that $T_{z}$ has resolvent $R_{z}$ which satisfies (13) for all $|z|<1$ and $x \in \mathscr{H}$. Then Lemma 2(b) implies $\left\|T_{z}\right\| \leqq 1,|z|<1, D\left(T_{z}\right)=\mathscr{C}$, which is (i). This proves Theorem $B$.

Corollary. $R_{z} \in \mathscr{R}, R_{0}=I$, and $N\left(R_{z}\right)=\{0\}$ for $|z|>1$ if and only if

$$
R_{z}=\left(I-z T_{z}\right)^{-1}, \quad|z| \neq 1
$$

where

(i) $\left\|T_{z}\right\| \leqq 1,|z|<1, D\left(T_{z}\right)=\mathscr{C}$.

(ii) $T_{z}$ is a holomorphic function of $z$ for $|z|<1$.

(iii) $T_{z^{*}}$ exists and $T_{z^{*}}^{-1}=T_{z}^{*}, 0<|z|<1$.

Proof. If $N\left(R_{z}\right)=\{0\},|z|>1$, then one may define

$$
T_{z} \equiv \frac{1}{z}\left(I-R_{z}^{-1}\right)
$$

for all $0<|z|<1$ and $|z|>1$. Then

$$
T_{z^{*}}=\bar{z}\left(I-R_{z^{*}}^{-1}\right)=\bar{z}\left(R_{z^{*}}-I\right) R_{z^{*}}^{-1}
$$

exists for $0<|z|<1$. Also, since $N\left(R_{z^{*}}-I\right)=N\left(R_{z}^{*}\right)=N\left(R_{z}\right)=\{0\}$ for $0<|z|<1$, $\left(R_{z^{*}}-I\right)^{-1}$ exists. Then $T_{z^{*}}^{-1}$ exists and

$$
\begin{aligned}
T_{z^{*}}^{-1} & =z^{*} R_{z^{*}}\left(R_{z^{*}}-I\right)^{-1}=z^{*}\left(R_{z}^{*}-I\right)\left(R_{z}^{*}\right)^{-1} \\
& =z^{*}\left[I-\left(R_{z}^{*}\right)^{-1}\right]=T_{z}^{*}, \quad 0<|z|<1 .
\end{aligned}
$$

Hence for $|z|>1$

$$
\begin{aligned}
R_{z} & =I-\left(I-z^{-1} T_{z^{*}}^{*}\right)^{-1}=I-\left(I-z^{-1} T_{z}^{-1}\right)^{-1} \\
& =I+\left[\left(I-z T_{z}\right) z^{-1} T_{z}^{-1}\right]^{-1}=I+z T_{z}\left(I-z T_{z}\right)^{-1} \\
& =I+\left(z T_{z}-I+I\right)\left(I-z T_{z}\right)^{-1}=I-I+\left(I-z T_{z}\right)^{-1} \\
& =\left(I-z T_{z}\right)^{-1} .
\end{aligned}
$$

Conversely, if $T_{z^{*}}$ exists and $R_{z}=\left(I-z T_{z}\right)^{-1},|z| \neq 1$, then

$$
R_{z}^{-1}=\left(I-z T_{z}\right)
$$

exists for $|z| \neq 1$. Therefore, $N\left(R_{z}\right)=\{0\}$ for all $|z| \neq 1$.

4. Triples related to unitary and partially unitary operators.

4.1. Consider the triple of classes $\mathscr{S}_{0}=\left\{\mathscr{E}_{0}, \mathscr{R}_{0}, \mathscr{T}_{0}\right\}$ defined as follows: 
$\left(\alpha_{0}\right) \quad E_{\theta}(0 \leqq \theta \leqq 2 \pi)$ belongs to the class $\mathscr{E}_{0}=\mathscr{E}_{0}(\mathscr{\mathscr { C }})$ whenever it is an orthogonal resolution of the identity, i.e., satisfies
(a) $E_{\theta}=E_{\theta}^{*}, 0 \leqq 0 \leqq 2 \pi$.
(b) $E_{\theta_{1}} E_{\theta_{2}}=E_{\min \left(\theta_{1}, \theta_{2}\right)}, \theta_{1}, \theta_{2} \in[0,2 \pi]$.
(c) $E_{\theta+0}=E_{\theta}, 0 \leqq \theta<2 \pi$.
(d) $E_{0}=0, E_{2 \pi}=I$.

( $\left.\beta_{0}\right) \quad R_{z}(|z| \neq 1)$ belongs to the class $\mathscr{R}_{0}=\mathscr{R}_{0}(\mathscr{H})$ whenever

(a) $z R_{z}-z^{\prime} R_{z^{\prime}}=\left(z-z^{\prime}\right) R_{z} R_{z^{\prime}},|z| \neq 1,\left|z^{\prime}\right| \neq 1$.

(b) If $R_{z} x=0$, then $x=0 . \quad(|z| \neq 1, x \in \mathscr{C})$.

(c) $R_{z}^{*}=I-R_{z^{*}}, z \neq 0$ and $|z| \neq 1$.

$\left(\gamma_{0}\right) \quad T^{(k)}( \pm k=0,1,2, \cdots)$ belongs to the class $\mathscr{T}_{0}=\mathscr{T}_{0}(\mathscr{H})$ whenever $T^{(1)} \equiv T$ is a unitary operator, and $T^{(k)} \equiv T^{k}, \pm k=0,1,2, \cdots$.

LEMma 3. Let $T$ be a unitary operator acting in $\mathscr{H}$. Then $T$ has associated with it a unique triple $\left\{E_{\theta}, R_{z}, T^{(k)}\right\}$ of functions in classes $\mathscr{E}_{0}, \mathscr{R}_{0}, \mathscr{T}_{0}$ respectively, determined as follows:

$\left(\alpha_{0}^{\prime}\right) \quad E_{\theta}$ is the spectral function of $T$, i.e.,

$$
T=\int_{0}^{2 \pi} e^{i \theta} d E_{\theta}
$$

( $\left.\beta_{0}^{\prime}\right) \quad R_{z}$ is the resolvent of $T$, i.e.,

$$
R_{z}=(I-z T)^{-1}, \quad|z| \neq 1 .
$$

$\left(\gamma_{0}^{\prime}\right) \quad T^{(k)}$ is the cyclic group of powers of $T$, i.e.,

$$
T^{(k)}=T^{k}, \quad \pm k=0,1,2, \cdots .
$$

Conversely, each function of class $\mathscr{E}_{0}, \mathscr{R}_{0}$, or $\mathscr{T}_{0}$ is associated with precisely one unitary $T$ in the manner just described.

Proof. These characterizations are elementary facts and their proofs will be omitted.

4.2. The functional correspondence (7) is multiplicative when $E_{\theta}$ is projector-valued, i.e., $E_{\theta}^{2}=E_{\theta}$. In the most general case $E_{2 \pi}$ is also a projector, but not necessarily the identity, i.e., $E_{2 \pi} \leqq I$. We have defined this class $\mathscr{E}_{1}$ in $\S 2.2$. Now define the triple of classes $\mathscr{S}_{1}=$ $\left\{\mathscr{E}_{1}, \mathscr{R}_{1}, \mathscr{T}_{1}\right\}$ of bounded linear operators as follows:

$\left(\alpha_{1}\right) \quad E_{\theta}(0 \leqq \theta \leqq 2 \pi)$ belongs to the class $\mathscr{E}_{1}=\mathscr{E}_{1}(\mathscr{C})$ whenever

(a) $E_{\theta}=E_{\theta}^{*}, 0 \leqq \theta \leqq 2 \pi$.

(b) $E_{\theta_{1}} E_{\theta_{2}}=E_{\min \left(\theta_{1}, \theta_{2}\right)}, \theta_{1}, \theta_{2} \in[0,2 \pi]$.

(c) $E_{\theta+0}=E_{\theta}, 0 \leqq \theta<2 \pi$.

(d) $E_{0}=0$. 
$\left(\beta_{1}\right) \quad R_{z}(|z| \neq 1)$ belongs to the class $\mathscr{R}_{1}=\mathscr{R}_{1}(\mathscr{H})$ whenever

(a) $z R_{z}-z^{\prime} R_{z^{\prime}}=\left(z-z^{\prime}\right) R_{z} R_{z^{\prime}},|z| \neq 1,\left|z^{\prime}\right| \neq 1$.

(b) $R_{z}^{*}=R_{0}-R_{z^{*}}, z \neq 0$ and $|z| \neq 1$.

$\left(\gamma_{1}\right) \quad T^{(k)}( \pm k=0,1,2, \cdots)$ belongs to the class $\mathscr{T}_{1}=\mathscr{T}_{1}(\mathscr{C})$ whenever $T^{(1)} \equiv T$ is a partially unitary operator, and $T^{(k)} \equiv T^{k}$, $\pm k=0,1,2, \cdots$; i.e.,

$$
T^{(k)}=T^{k}=U^{k} \oplus 0, \quad \pm k=0,1,2, \cdots,
$$

where $\mathscr{Y}=\mathscr{K}_{U} \oplus \mathscr{\mathscr { C }}{ }_{0}, U$ is a unitary operator on $\mathscr{C}_{U}$, and 0 is the zero operator on $\mathscr{C}_{0}$.

REMARKs. (i) The class $\mathscr{R}_{1}$ is similar to the class of pseudoresolvents discussed by Hille and Phillips [6, §5.8-5.10].

(ii) $T^{(k)} \in \mathscr{T}_{1}$ satisfies

$$
\begin{aligned}
& \text { (a) } T^{(k) *}=T^{(-k)}, \pm k=0,1,2, \cdots \\
& \text { (b) } T^{(k)} T^{(m)}=T^{(k+m)}, \pm k, \pm m=0,1,2, \cdots
\end{aligned}
$$

\section{Lemma 4. $\mathscr{S}_{0} \subset \mathscr{S}_{1} \subset \mathscr{S}$.}

Proof. The only new part of this lemma is $\mathscr{R}_{1} \subset \mathscr{R}$. Suppose $R_{z} \in \mathscr{R}_{1}$. $\quad\left(\beta_{1}\right.$ a) implies that $R_{z}$ is a holomorphic function of $z$ for $|z| \neq 1$. Note that $\operatorname{Re}\left(R_{z} x, x\right)=\frac{1}{2}\left[\left(R_{z} x, x\right)+\left(R_{z}^{*} x, x\right)\right]$. If $0<|z|<$ 1 , or $|z|>1$, then successively substituting $\left(\beta_{1} b\right)$ for $R_{z}^{*}$ and $\left(\beta_{1} a\right)$ for $R_{z^{*}}$ in this equality, and simplifying, we obtain

$$
\operatorname{Re}\left(R_{z} x, x\right)=\frac{1}{2}\left(R_{0} x, x\right)+\frac{1}{2}\left(1-|z|^{2}\right)\left\|R_{z} x\right\|^{2} .
$$

Furthermore, this equality shows that $\left(R_{0} x, x\right)$ is real, hence $R_{0}=R_{0}^{*}$. Also, $\left(\beta_{1} \mathrm{a}\right)$ for $z^{\prime}=0$ and the continuity of $R_{z}$ at $z=0$ implies $R_{0}=R_{0}^{2}$. Then $\left\|R_{0} x\right\|^{2}=\left(R_{0}^{2} x, x\right)=\left(R_{0} x, x\right)$, thus the inequality in $(\beta b)$ is an equality for all $|z| \neq 1$. Hence $R_{z} \in \mathscr{R}$.

4.3 Theorem $\mathrm{A}_{0}$. The triple of classes $\mathscr{S}_{0}=\left\{\mathscr{E}_{0}, \mathscr{R}_{0}, \mathscr{T}_{0}\right\}$ is a matched triple of classes.

Proof. We need to show that $\mathscr{S}_{0}$ satisfies the requirements (i) and (ii) of the definition of a matched triple of classes given in $\S 1$. But (i) was proved in Lemma 4, and (ii) follows from Lemma 3.

Theorem $\mathrm{A}_{1}$. The triple of classes $\mathscr{S}_{1}=\left\{\mathscr{E}_{1}, \mathscr{R}_{1}, \mathscr{T}_{1}\right\}$ is a matched triple of classes.

Proof. By Lemma $4 \mathscr{S}_{1} \subset \mathscr{S}_{\text {. }}$ In Theorem 3 of $\S 3.2$ it was shown 
that $N=N\left(E_{2 \pi}\right)=N\left(T^{(0)}\right)=N\left(R_{z}\right)$ for $|z|<1$, and that the decomposition $\mathscr{H}=N^{\perp} \oplus N$ is reducing for all values of $E_{\theta}, R_{z}$, and $T^{(k)}$. By Theorem $A_{0}, \mathscr{S}_{1}\left(N^{\perp}\right)$ is a matched triple of classes, and trivially, $S_{1}(N)$ is a matched triple of classes. It follows that $\mathscr{S}_{1}(\mathscr{H})$ is a matched triple of classes.

4.4. The following theorem asserts the existence of a type $\mathscr{S}_{1}$ minimal dilation of an arbitrary matched triple of functions.

Theorem C. Let $\left\{E_{\theta}, R_{z}, T^{(k)}\right\} \in \mathscr{S}(\mathscr{H})$ be a matched triple of functions. There exists a Hilbert space $\mathscr{Y}^{+} \supseteqq \mathscr{\mathscr { C }}$ and a matched triple of functions $\left\{E_{\theta}^{+}, R_{z}^{+}, T^{(k)+}\right\} \in \mathscr{S}_{1}\left(\mathscr{C}^{+}\right)$such that

(i) for $x \in \mathscr{H}$

$$
E_{\theta} x=P E_{\theta}^{+} x, \quad R_{z} x=P R_{z}^{+} x, \quad T^{(k)} x=P T^{(k)+} x,
$$

where $P$ is the orthogonal projector onto the subspace $\mathscr{H}$ of $\mathscr{H}^{+}$.

(ii) $\mathscr{H}^{+}=\operatorname{span}\left(\mathscr{H}, \mathscr{C}_{0}\right)$, where

$$
\begin{aligned}
\mathscr{C}_{0} & =\operatorname{span}\left\{E_{\theta}^{+} x: x \in \mathscr{H}, 0 \leqq \theta \leqq 2 \pi\right\} \\
& =\operatorname{span}\left\{R_{z}^{+} x: x \in \mathscr{C},|z| \neq 1\right\} \\
& =\operatorname{span}\left\{T^{(k)+} x: x \in \mathscr{H}, \pm k=0,1,2, \cdots\right\} .
\end{aligned}
$$

(iii) $E^{+}(\sigma)=0$ if and only if $E(\sigma)=0$, where $\sigma$ is any Borel set on $[0,2 \pi]$ and $E(\sigma)$ is the operator measure related to $E_{\theta}$. Also, $E_{2 \pi}^{+}=I$ if and only if $E_{2 \pi}=I$.

Proof. The assertions involving $E_{\theta}$ are contained in the Naimark diation theorem. The other two relations in (i) follow immediately from Theorems $A$ and $A_{1}$. The equivalence of the spans in (ii) follows from formulas (1)-(6).

Note that in the special case of $T^{(k)}=T^{k}$ for $k \geqq 0$ and $T^{(k)}=$ $\left(T^{*}\right)^{|k|}$ for $k<0$, Theorem C contains the fundamental Sz.-Nagy Unitary Dilation Theorem [17, Th. 1] and [16, Th. III]. Also, for arbitrary $\left\{E_{0}, R_{z}, T^{(k)}\right\} \in \mathscr{S}$, the minimal dilation $\left\{E_{\theta}^{+}, R_{z}^{+}, T^{(k)+}\right\} \in \mathscr{S}_{1}$ will be proper if and only if $E_{2 \pi}=I$. In particular, a proper triple need not have a proper minimal dilation.

\section{Triples related to contraction operators.}

5.1 THEOREM 4. The following conditions on a bounded operatorvalued function $R_{z}$ acting on $\mathscr{C}$ are equivalent:

$$
\begin{aligned}
R_{z} & =\sum_{k=0}^{\infty} z^{k} T^{k} \quad(|z|<1) \\
& =-\sum_{k=1}^{\infty} z^{-k} T^{* k} \quad(|z|>1)
\end{aligned}
$$


where $T$ is a fixed contraction operator on $\mathscr{C}$.

$$
\begin{aligned}
R_{z} & \left.=(I-z T)^{-1} \quad(|z|)<1\right) \\
& =I-\left(I-z^{-1} T^{*}\right)^{-1} \quad(|z|>1)
\end{aligned}
$$

where $T$ is a fixed contraction operator on $\mathscr{H}$.

$$
\begin{aligned}
& R_{z}=(I-z T)^{-1} \quad\left(\left|z^{*}\right|<1\right) \\
& \quad=I-\left(I-z^{-1} T^{*}\right)^{-1} \quad(|z|>1),
\end{aligned}
$$

and

$$
(1-|z|)\left\|R_{z}-I\right\| \leqq|z| \quad\left(|z|_{i}^{*}<1\right),
$$

where $T$ is some fixed operator with $\overline{D_{T}}=\mathscr{C}$.

(iv) (a) $z R_{z}-\zeta R_{\zeta}=(z-\zeta) R_{z} R_{\zeta}$ for $|z|,|\zeta|<1$.

(b) $\quad(1-|z|)|| R_{z}-I|| \leqq|z|, \quad|z|<1$.

(c) $\quad R_{z^{*}}=I-R_{z}^{*}, \quad 0<|z|<1$.

Under these conditions $R_{z} \in \mathscr{R}$ and $R_{0}=I$.

Proof. (i) $\Leftrightarrow$ (ii): This follows by showing that the series in (i) are the expansions of the resolvents in (ii) (cf. [20, p. 261]).

(ii) $\Leftrightarrow$ (iii): That (ii) implies (iii) follows from Lemma 2 (a). Assume (iii). The inequality for $z=0$ gives $R_{0}=I$. Define

$$
T(z) \equiv \frac{1}{z}\left(R_{z}-I\right), \quad 0<|z|<1,
$$

and

$$
T(0) \equiv \operatorname{Lim}_{z \rightarrow 0} T(z)
$$

Then for $x \in \mathscr{H}$.

$$
T(z) x=\frac{1}{z}\left(R_{z} x-I x\right)=\frac{1}{z}\left[R_{z} x-R_{z}(I-z T) x\right]=" R_{z} T x .
$$

Hence

$$
T(0) x=\operatorname{Lim}_{z \rightarrow 0} R_{z} T x=R_{0} T x=\frac{5}{z} T x .
$$

Using the inequality in (iii) we obtain

$$
\|T(z) x\|=\frac{1}{|z|}\left\|\left[R_{z}-I\right] x\right\| \leqq \frac{1}{1-|z|}\|x\|,
$$

for $|z|<1, x \in \mathscr{H}$. In particular,

$$
\|T x\|=\|T(0) x\| \leqq\|x\|,
$$

for all $x \in \mathscr{C}$, i.e., $T$ is a contraction operator on $\mathscr{H}$.

(iii) $\Leftrightarrow$ (iv): It is easy to show that $R_{z}=(I-z T)^{-1},|z|<1$, 
satisfies the resolvent equation (iva). Conversely, (iva) implies $R_{z}^{-1}$ exists for all $|z|<1$. Therefore, one may define $T=\left(I-R_{z}^{-1}\right) / z$, $0<|z|<1$, where (iva) implies that $T$ is independent of $z$. Then $R_{z}=(I-z T)^{-1},|z|<1$. By taking adjoints we obtain the equivalence of (iva) and the form of $R_{z}$ in (iii) for $|z|>1$.

Condition (ii) and Theorem $\mathrm{B}$ imply $R_{z} \in \mathscr{R}$ and $R_{0}=I$.

CoROLlary. If $T$ is a contraction operator on $\mathscr{H}$, and $T^{(k)}$ is defined by $T^{(k)}=T^{k}$ for $k=0,1,2, \cdots$, and $T^{(k)}=\left(T^{*}\right)^{|k|}$ for $k=$ $-1,-2, \cdots$, then $T^{(k)} \in \mathscr{T}$.

Proof. If $T^{(k)}$ is defined as in the statement of the corollary, then the corresponding $R_{z}$ defined by formula (3) satisfies condition (i) of the theorem. Therefore, $R_{z} \in \mathscr{R}$, and $T^{(k)} \in \mathscr{T}$ by Theorem A.

REMARKs. 1. A direct proof of the above corollary has been given previously by Sz.-Nagy [16, §9].

2 . Under the conditions of Theorem $4, \mathrm{R}_{z}$ also satisfies the resolvent equation (iva) for $|z|,|\zeta|>1$. Then $N\left(R_{z}\right)$ is constant for $|z|>1$, but in general, we do not have $N\left(R_{z}\right)=\{0\}$ for $|z|>1$.

3. If the resolvent equation (iva) is satisfied for $z$ and $\zeta$ on opposite sides of the unit circle, i.e., for all $|z|,|\zeta| \neq 1$, then $N\left(R_{z}\right)=$ $\{0\}$ for all $|z| \neq \neq 1$, and $R_{z} \in \mathscr{R}_{0}$. In this case $R_{z}=(I-z T)^{-1}$ for all $|z| \neq 1$, where $T$ is necessarily unitary by Lemma 3 of 3.1 .

5.2. Using the terminology of M. Schreiber $[12,13]$, we make the following:

Definition. An operator distribution function $E_{j} \in B$ is called strong (or Nagy) if

$$
\begin{aligned}
\int_{0}^{2 \pi} e^{i k \theta} d E_{\theta}=\left[\int_{0}^{2 \pi} e^{i \theta} d E_{\vartheta}\right]^{k}, \quad k & =0,1,2, \cdots \\
= & {\left[\int_{0}^{2 \pi} e^{i \vartheta} d E_{0}\right]^{* i k \mid}, \quad k=-1,-2, \cdots . }
\end{aligned}
$$

REMARK. Necessarily $E_{2 \pi}=I$ for strong operator distribution functions.

We consider the classes $\mathscr{E}_{2}, \mathscr{R}_{2}$, and $\mathscr{T}_{2}$ defined as follows:

$\left(\alpha_{2}\right) \quad E_{\theta} \quad(0 \leqq \theta \leqq 2 \pi)$ belongs to the class $\mathscr{E}_{2}=\mathscr{E}_{2}(\mathscr{C})$ whenever $E_{\theta} \in \mathscr{E}$ is a strong operator distribution function.

$\left(\beta_{2}\right) \quad R_{z}(|z| \neq 1)$ belongs to the class $\mathscr{R}_{2}=\mathscr{R}_{2}(\mathscr{C})$ whenever (a) $z R_{z}-\zeta R_{\zeta}=(z-\zeta) R_{z} R_{\zeta}$ for $|z|,|\zeta|<1$. 
(b) $(1-|z|)|| R_{z}-I|| \leqq|z|,|z|<1$.

(c) $\quad R_{z^{*}}=I-R_{z}^{*}, 0<|z|<1$.

$\left(\gamma_{2}\right) T^{(k)}( \pm k=0,1,2, \cdots)$ belongs to the class $\mathscr{T}_{2}=\mathscr{T}_{2}(\mathscr{C})$ whenever $T^{(1)} \equiv T$ is a contraction operator on $\mathscr{\mathscr { C }}$ and

$$
\begin{aligned}
T^{(k)} & \equiv T^{k}, \quad k=0,1,2, \cdots \\
& \equiv T^{*|k|}, \quad k=-1,-2, \cdots .
\end{aligned}
$$

Theorem D. The triple of classes $\mathscr{S}_{2}=\left\{\mathscr{E}_{2}, \mathscr{R}_{2}, \mathscr{T}_{2}\right\}$ is a matched triple of classes.

Proof. (i ) $\mathscr{S}_{2} \subset \mathscr{S}$ : Suppose $\left\{E_{\theta}, R_{z}, T^{(k)}\right\} \in \mathscr{S}_{2}$. By $\left(\alpha_{2}\right) E_{\theta} \in \mathscr{E}$. Theorem 4 implies $R_{z} \in \mathscr{R}$ and its corollary implies $T^{(k)} \in \mathscr{T}$. Therefore, $\left\{E_{\theta}, R_{z}, T^{(k)}\right\} \in \mathscr{S}$. It is clear that $\mathscr{S}_{2} \neq \mathscr{S}$, so $\mathscr{S}_{2} \subset \mathscr{S}$.

(ii) If $\left\{E_{0}, R_{z}, T^{(k)}\right\} \in \mathscr{S}$, then any one of the relations $E_{\theta} \in \mathscr{E}_{2}$, $R_{z} \in \mathscr{R}_{2}, T^{\langle k\rangle} \in \mathscr{T}_{2}$ implies all three, i.e., implies that $\left\{E_{\theta}, R_{z}, T^{(k)}\right\} \in \mathscr{S}$ :

$E_{\theta} \in \mathscr{E}_{2} \Leftrightarrow T^{(k)} \in \mathscr{T}_{2}$ is established by Sz.-Nagy in [17, p. 90, (3)(5)]. See also [12, Th. 2.2] and [3, Th. 4].

If $T^{(k)} \in \mathscr{T}_{2}$, then the corresponding $R_{z}$ defined by formula (3) satisfies condition (i) of Theorem 4, which is equivalent to the conditions of $\left(\beta_{2}\right)$. Hence $R_{z} \in \mathscr{R}_{2}$. Conversely, if $R_{z} \in \mathscr{R}_{2}$, then equating coefficients in the series of formula (3) and Theorem 4(i), we see that $T^{(k)}$ is of the necessary form for $T^{(k)} \in \mathscr{T}_{2}$. This proves Theorem D.

We remark that the new part of Theorem $D$ is the characterization $\left(\beta_{2}\right)$ of the interrelated resolvent class $\mathscr{R}_{2}$. We also note that $\mathscr{S}_{0} \subset \mathscr{S}_{2} \subset \mathscr{S}_{\text {. }}$

5.3. In general, the functional correspondence (7) is not multiplicative for $f \in B(d E)$. However, if we require $E_{\theta} \in \mathscr{E}_{2}$, then for certain subclasses of $B(d E)$ the functional calculus is multiplicative.

Definition. The Hardy $H_{\infty}$ class of functions is the algebra of bounded holomorphic functions in the unit disc [7].

By Fatou's theorem [7, p. 34] the limit $f\left(e^{i \theta}\right)$ of $f\left(r e^{i \theta}\right)$ as $r \rightarrow 1^{-}$ exists almost everywhere with respect to $\theta$, i.e., everywhere except on a set $C_{f}$ of Lebesgue measure zero.

Definition. By $H_{\infty}(d E)$ we mean the subalgebra of $H_{\infty}$ such that

$$
E\left(C_{f}\right)=\int_{0}^{2 \pi} \chi\left(C_{f} ; \theta\right) d E_{\theta}=0,
$$

where $C_{f}$ is that set of Lebesgue measure zero given in Fatou's theorem, $\chi\left(C_{f} ; \theta\right)$ is the characteristic function of $C_{f}$, and $E_{0} \in \mathscr{E}_{2}$. 
Note that $H_{\infty}(d E) \subset B(d E)$ since the above definition implies that the set $C_{f}$ does not belong to $\Lambda(E)$.

Assuming $E_{\theta} \in \mathscr{E}_{2}$, the functional correspondence given in $\S 2.3$ has the following additional properties (cf. [19]):

( $\mathrm{x}$ ) If $f, g$ are polynomials in $e^{i \theta}$ (or in $e^{-i \theta}$ ), then $f g \sim F G$.

(xi ) $f_{n} \rightarrow f$ and $g_{n} \rightarrow g$ boundedly a.e. $(d E)$ and $f_{n} g_{n} \sim F_{n} G_{n}$ implies $f g \sim F G$.

(xii) When $f, g \in H_{\infty}(d E)$, then $f g \sim F G$.

(xiii) If $f \in H_{\infty}(d E)$, then $f \sim F=F(T), T$ a contraction operator, and

$$
\|F(T)\| \leqq \sup _{|z|<1}|f(z)|
$$

It follows that the mapping $f \rightarrow F$ given by (7) is a homomorphism from the algebra $H_{\infty}(d E)$ into the algebra of bounded linear transformations of the Hilbert space $\mathscr{H}$. Further properties of this mapping have been studied by Schreiber [13] and Sz.-Nagy [18, 19].

\section{The isometric operator case.}

6.1. Let $R_{z} \in \mathscr{R}$ and suppose that for a certain operator $T$ the relation

$$
R_{z}(I-z T) x=x \quad\left(x \in D_{T}\right)
$$

holds for some $z$ such that $|z| \neq 1$.

Remarks. ( i ) Formula (17) is equivalent to the statement that $(I-z T)$ has a bounded inverse satisfying $(I-z T)^{-1} \subseteq R_{z}$.

(ii) If $R_{0}=I$ and (17) is valid for any point $z_{0}$ such that $0<\left|z_{0}\right|<1$, then $\|T x\| \leqq\|x\|$ for all $x \in D_{T}$, i.e., $\|T\| \leqq 1$. Furthermore, if $R_{0}=I$ and (17) is valid for any point $z_{0}$ such that $\left|z_{0}\right|>1$, then $\|T x\| \geqq\|x\|$ for all $x \in D_{T}$.

(iii) If $R_{0}=I$ and (17) holds for any two points $z_{1}, z_{2}$ such that $0<\left|z_{1}\right|<1$ and $\left|z_{2}\right|>1$, then $T$ is isometric.

(iv) If $R_{z} \in \mathscr{R}$ and $R_{0}=I$, then relation (17) is equivalent to $T \subseteq T_{z},|z|<1$; and $T^{-1} \subseteq T_{z^{*}}^{*},|z|>1$, where $T_{z}$ and $T_{z^{*}}^{*}$ are the operators introduced in Theorem B.

(v) If $R_{z} \in \mathscr{R}, R_{0}=I$, and $N\left(R_{z}\right)=\{0\}$ for $|z|>1$, then relation (17) is equivalent to $T \subseteq T_{z},|z| \neq 1$, where $T_{z}$ is as in the corollary of Theorem B.

(vi) If relation (17) holds in an open set contained in $|z|<1$ (or $|z|>1$ ), then by analytical continuation it holds throughout $|z|<1$ (or $|z|>1$ ).

(vii) If relation (17) holds for $|z|<1$, then $E_{2 \pi} x=x$ for $x \in \overline{D_{T}}$. 
In particular, if $\overline{D_{r}}=\mathscr{H}$, then $E_{2 \pi}=I$.

Proof. (i) is clear. (ii) follows by substituting (17) into formulas $\left(13^{\prime}\right)$ and $\left(13^{\prime \prime}\right)$. (iii) follows from (ii) since an operator $T$ is isometric if and only if $\|T x\|=\|x\|$ for all $x \in D_{T}$ [14, Th. 2.46]. Property (iv) follows from the resolvent representations in Theorem B. For $|z|<1$ we may substitute $R_{z}=\left(I-z T_{z}\right)^{-1}$ into (17) and obtain the equivalent equation $T x=T_{z} x, x \in D_{T}$. For $|z|>1$, we may substitute $R_{z}=I-\left(I-z^{-1} T_{z^{*}}^{*}\right)^{-1}$ into (17) and obtain the equivalent equation $I x=T_{z^{*}}^{*} T x, x \in D_{T}$, which is equivalent to $T$ having an inverse satisfying $T^{-1} \cong T_{z^{*}}^{*}$. Property (v) is obtained from (iv) and the corollary to Theorem B. (vi) is clear. (vii) follows by letting $z=0$ in (17) and using Remark (i) of $\S 3.1$.

Theorem E 6.2. Suppose that $\left.\left\{E_{0}, R_{z}, T^{k}\right)\right\}$ is a matched triple of functions in $S$ with $E_{2 \pi}=I$, that $R_{z}$ is represented by $T_{z}$, and that $E_{\theta}^{+}$is the minimal dilation of $E_{\theta}$. Let $T$ be a certain operator with $D_{T} \subseteq \mathscr{H}$. Then these conditions are equivalent:

(i) $T$ is an isometric operator such that $T \cong T^{11}$.

$$
T \subseteq T^{(1)+}=\int_{0}^{2 \pi} e^{i \theta} d E_{\theta}^{+} .
$$

(iii) $R_{z}(I-z T) x=x$, for $|z| \neq 1$ and $x \in D_{T}$.

(iv) $T \cong T_{z}$ for $|z|<1, T^{-1}$ exists, and $T^{-1} \cong T_{z^{*}}^{*}$ for $|z|>1$.

Proof. ( i ) $\Leftrightarrow$ (ii): Assume (i). Then $T x=T^{(1)} x=P T^{(1)}+x$ for $x \in D_{T}$. But for $x \in D_{T}$

$$
\|T x\|=\|x\|=\left\|T^{(1)+} x\right\|,
$$

since $T$ is isometric, and $T^{(1)+}$ is unitary by Lemma 3 . Therefore, $T x=T^{(1)+} x$ for all $x \in D_{T}$, i.e., $T \subseteq T^{(1)+}$.

Suppose (ii). $\quad T \subseteq T^{(1)+}$ implies $T x=T^{(1)+} x$ for all $x \in D_{T}$. Then by property (v) of $\S 2.3$ and equation (9)

$$
\begin{aligned}
\|T x\|^{2} & =\left\|T^{(1)+} x\right\|^{2}=\int_{0}^{2 \pi}\left|e^{i \theta}\right|^{2} d\left(E_{0}^{+} x, x\right)=\int_{0}^{2 \pi} d\left(E_{\theta}^{+} x, x\right)=\left(E_{2 \pi}^{+} x, x\right) \\
& =\left(E_{2 \pi} x, x\right)=\|x\|^{2} \quad \text { for all } x \in D_{T^{\prime}},
\end{aligned}
$$

i.e., $T$ is an isometric operator. Also, $T \subseteq T^{(1)+}$ implies

$$
T x=T^{(1)+} x=P T^{(1)+} x_{\alpha}=T^{(1)} x
$$

for $x \in D_{T}$, i.e., $T \cong T^{(1)}$.

(ii) $\Longrightarrow$ (iii): Since $E_{\theta}^{+} \in \mathscr{E}_{0}$, Lemma 3 implies that $T^{(1)+}$ is unitary and $R_{z}=\left(I-z T^{(1)+}\right)^{-1},|z| \neq 1$. Therefore, 


$$
R_{z}\left(I-z T^{(1)+}\right) x=x
$$

for all $x \in \mathscr{X}$, and in particular, for all $x \in D_{T}$. But for $x \in D_{T}$, $T^{(1)}+x=T x$ by (ii), hence this becomes (iii).

(iii) $\Rightarrow$ (ii): For $x \in D_{T}$, set $y=(I-z T) x$. Using (iii) and an argument similar to $[8, \S 5.2]$ one may show successively that $R_{z}$ satisfies the resolvent equation, $R_{z^{*}} R_{z} y=P R_{z^{+}}^{+} R_{z}^{+} y$, and $R_{z} y=R_{z}^{+} y$ for all $y \in \Delta_{T}(z)$. Then $T x=T^{(1)+} x$ for all $x \in D_{T}$, i.e., $T \subseteq T^{(1)+}$.

(iii) $\Leftrightarrow$ (iv): This follows from Remark (iv) of $\S 6.1$.

REMARKs. 1. When $\overline{\Delta_{T}}=\mathscr{C}$, condition (iv) of Theorem $\mathrm{E}$ takes the form

(iv') $T^{-1}$ exists, and $T \subseteq T_{z}=\left(T^{-1}\right)^{*}$, for $|z|<1$.

2. When $\overline{D_{T}}=\mathscr{C}$, condition (iv) of Theorem $\mathrm{E}$ takes the form (iv") $T^{-1}$ exists, and $T^{-1} \subseteq T_{z}^{*}=T^{*}$, for $|z|<1$.

3. By Remark (vii) of $\S 6.1$, the condition $E_{2 \pi}=I$ in Theorem $\mathrm{E}$ may be omitted in (iv"), and weakened in parts (iii) and (iv) to $E_{2 \pi} x=x$ for all $x \in \mathscr{L} \ominus \overline{D_{T}}$.

Condition (iii) (or (iv)) of Theorem $\mathrm{E}$ characterizes those $R_{z} \in \mathscr{R}$ with $R_{0}=I$ which are generalized resolvents of a given isometric operator T. Using Remark 3, we obtain the following:

CoRollary. In order that a set of bounded linear operators $R_{z}$ in $\mathscr{C}$ (with $D\left(R_{z}\right)=\mathscr{C}$ and $|z| \neq 1$ ) be a generalized resolvent of the isometric oprrator $T$ in $\mathscr{C}$, it is necessary and sufficient that the following conditions be satisfied:

(a) $R_{z} \in \mathscr{R}=\mathscr{R}(\mathscr{C}),|z| \neq 1$

(b) $R_{z}(I-z T) x=x$, for all $x \in D_{T}$ and $|z| \neq 1$

(c) $R_{0} x=x$ for all $x \in \mathscr{H} \ominus \overline{D_{T}}$.

We remark that for $T$ a closed isometric operator, this corollary is equivalent to Theorem 2 of [4]. The generalized resolvent of a closed isometric operator $T$ was defined in [4] as a set $R_{z}(|z| \neq 1)$ of operators in $\mathscr{H}$ satisfying

$$
R_{z}=P\left(I-z T^{+}\right)^{-1} x, \quad x \in \mathscr{C},
$$

where $T^{+}$is a unitary extension of $T$ in a Hilbert space $\mathscr{C}^{+} \supseteqq \mathscr{C}^{2}$, and $P$ is the orthogonal projection of $\mathscr{C}^{+}$onto $\mathscr{C}$. We note that Theorem $\mathrm{C}$ implies that this definition is equivalent to the conditions given in the corollary.

6.3 Condition (iv) of Theorem $\mathrm{E}$ leads to a simple and direct proof of a formula for all generalized resolvents of a closed isometric operator 
which is analogous to Štraus's formula for symmetric operators [15, Th. 7]. The formula was announced by M. E. Cumakin, a student of Štraus, in 1964 [4, Th. 3]

We shall need the following definitions and lemma which were originally introduced by McKelvey in [8].

Definition. A pair of operators $L_{1}, L_{2}$ acting in $\mathscr{\mathscr { C }}$ are said to be formal adjoints whenever

$$
\left(L_{1} x, y\right)=\left(x, L_{2} y\right)
$$

for $x \in D\left(L_{1}\right), y \in D\left(L_{2}\right)$.

DEFINITION. A pair of formally adjoint contraction operators $B_{+}, B_{-}$ will be called a *-pair between closed subspaces $\mathscr{H}_{+}, \mathscr{C}_{-}$of $\mathscr{C}$ whenever

$$
D\left(B_{ \pm}\right)=\mathscr{H}_{ \pm}, \quad B_{ \pm} \mathscr{H}_{ \pm} \subseteq \mathscr{H}_{\mp} .
$$

Definition. A *-pair $\widetilde{B}_{+}, \widetilde{B}_{-}$will be called a maximal *-extension of the *-pair $B_{+}, B_{-}$whenever

$$
\widetilde{B}_{ \pm} \supseteqq B_{ \pm} \text {and } D\left(\widetilde{B}_{ \pm}\right)=\mathscr{Y} \text {. }
$$

Lemma 5. Let $B_{+}, B_{-}$be a given *-pair between the subspaces $\mathscr{H}_{+}, \mathscr{H}_{-}$, and let $B_{+}^{\prime}, B_{-}^{\prime}$ be any *-pair between the subspaces $\mathscr{H} \ominus \mathscr{H}_{+}, \mathscr{H} \ominus \mathscr{H}_{-}$. Then

$$
\widetilde{B}_{ \pm}=B_{ \pm} \oplus B_{ \pm}^{\prime}
$$

is a maximal *-extension of $B_{+}, B_{-}$. Conversely, every maximal *-extension of $B_{+}, B_{-}$has this form.

Suppose that $T$ is a closed isometric operator acting in $\mathscr{C}$. Then $D_{T}=\overline{D_{T}} \cong \mathscr{H}$. Set $\mathscr{H}_{+}=D_{T}$ and $\mathscr{H}_{-}=\Delta_{T}$. Then $\mathscr{H}_{-}=T \mathscr{C}_{+}$and $\mathscr{H}_{+}=T^{-1} \mathscr{H}_{-}$.

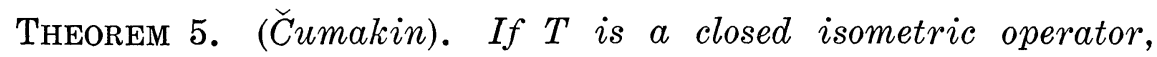
then a necessary and sufficient condition that the operator-valued functions $T_{z},|z|<1$, and $T_{z^{*}}^{*} x,|z|>1$, shall represent a generalized resolvent $R_{z}$ of $T$ in the sense of Theorem $E(i v)$ is that

$$
T_{z}=T \oplus \Phi_{+}(z), \quad|z|<1
$$

and

$$
T_{z}^{*}=T^{-1} \oplus \Phi_{-}(z), \quad|z|<1,
$$


where

(a) $\Phi_{ \pm}(z)$ is a formally adjoint pair of linear operators mapping $\mathscr{H} \ominus \mathscr{H}_{ \pm}$into $\mathscr{H} \ominus \mathscr{H}_{\mp}$.

(b) $\left\|\Phi_{ \pm}(z)\right\| \leqq 1$.

(c) $\Phi_{+}(z)$ is a holomorphic operator-valued function of $z$ for $|z|<1$, and $\Phi_{-}\left(z^{*}\right)$ is a holomorphic operator-valued function of $z$ for $|z|>1$.

Proof. Note that $T, T^{-1}$ form a *-pair between the closed subspaces $\mathscr{H}_{+}, \mathscr{H}_{-}$.

Suppose $T_{z},|z|<1$, and $T_{z^{*}}^{*},|z|>1$, represent a generalized resolvent of $T$, i.e., $T \subseteq T_{z},|z|<1$, and $T^{-1} \cong T_{z^{*}}^{*},|z|>1$. Recall that the second condition is equivalent to $T^{-1} \subseteq T_{z}^{*},|z|<1$. Now $T_{z}$ and its adjoint $T_{z}^{*},|z|<1$, form a maximal *-extension of $T, T^{-1}$. By Lemma $5, T_{z}$ and $T_{z}^{*}$ have the form

$$
T_{z}=T \oplus \Phi_{+}(z) \quad \text { and } \quad T_{z}^{*}=T^{-1} \oplus \Phi_{-}(z),
$$

where $\Phi_{+}(z), \Phi_{-}(z)$ is a ${ }^{*}$-pair between the subspaces $\mathscr{H} \ominus \mathscr{H}_{+}$, and $\mathscr{H} \ominus \mathscr{H}_{-}$. Then (a) and (b) are satisfied. Now $\Phi_{+}(z)$ is holomorphic for $|z|<1$ because $T_{z}$ is, and $\Phi_{-}\left(z^{*}\right)$ is holomorphic for $|z|>1$ because $T_{z^{*}}^{*}$ is, i.e., (c) is also fulfilled.

Conversely, suppose

$$
T_{z}=T \oplus \Phi_{+}(z) \quad \text { and } \quad T_{z}^{*}=T^{-1} \oplus \Phi_{-}(z),
$$

for $|z|<1$, where $\Phi_{ \pm}(z)$ satisfy (a)-(c). By Lemma 5, $T_{z}, T_{z}^{*}$ form a maximal *-extension of $T, T^{-1}$. Then $T \subseteq T_{z}$ and $T^{-1} \subseteq T_{z}^{*}$, for $|z|<1$; hence $T^{-1} \subseteq T_{z^{*}}^{*},|z|>1$. Also, (c) implies $T_{z}$ is holomorphic for $|z|<1$, and $T_{z^{*}}^{*}$ is holomorphic for $|z|>1$. Therefore, $T_{z},|z|<1$, and $T_{z^{*}}^{*},|z|>1$, represent a generalized resolvent $R_{z}$ of $T$. This proves Theorem 5 .

The author wishes to express his sincere appreciation to Professor R. W. McKelvey for his guidance and encouragement in this work.

\section{BIBLIOGRAPHY}

1. N. I. Akhiezer and I. M. Glazman, Theory of linear operators in Hilbert space, Vol. 1, Ungar, New York, 1961.

2. - Theory of linear operators in Hilbert space, Vol. 2, Ungar, New York, 1963.

3. S. K. Berberian, Naǐmark's moment theorem, Michigan Math. J. 13 (1966) 171-184.

4. M. E. Cǔmakin, Generalized resolvents of an isometric operator, Dokl. Akad. Nauk SSSR 154 (1964), 791-794; Soviet Math. Dokl. 5 (1964), 193-196.

5. G. Herglotz, Über potenzreihen mit positivem, reellem teil im einheitskreis, Ber. Sächs. Ges. d. Wiss. Leipzig 63 (1911), 501-511.

6. E. Hille and R. S. Phillips, Functional analysis and semi-groups, revised ed., 
Amer. Math. Soc. Colloq. Pub. vol. 31, R. I., 1957.

7. K. Hoffman, Banach spaces of analytic functions, Prentice-Hall, N. J., 1962.

8. R. McKelvey, Spectral measures, generalized resolvents, and functions of positive type, J. Math. Anal. Appl. 11 (1965), 447-477.

9. F. Riesz, Sur certains systèmes singuliers d'équations intégrales, Ann. Sci. École Norm. Sup. (3) 28 (1911), 33-62.

10. F. Riesz and B. Sz.-Nagy, Functional analysis, Ungar, New York, 1955.

11. H. L. Royden, Real analysis, Macmillan, New York, 1963.

12. M. Schreiber, Unitary dilations of operators, Duke Math. J. 23 (1956), 579-594.

13. - A functional calculus for general operators in Hilbert space, Trans. Amer. Math. Soc. 87 (1958), 108-118.

14. M. H. Stone, Linear transformations in Hilbert space, Amer. Math. Soc. Colloq. Pub. vol. 15, New York, 1932.

15. A. V. Štraus, Generalized resolvents of symmetric operators, Izv. Akad. Nauk SSSR Ser. Mat. 18 (1954) 51-86.

16. B. Sz.-Nagy, Extensions of linear transformations in Hilbert space Which Extend Beyond This Space, Ungar, New York, 1960 (Appendix to [10]).

17. - Sur les contractions de l'espace de Hilbert, Acta Sci. Math. Szeged 15 (1953), 87-92.

18. B. Sz.-Nagy and C. Foias, Sur les contractions de l'espace de Hilbert, III, Acta Sci. Math. Szeged 19 (1958), 26-45.

19. B. Sz.-Nagy and C. Foias, Sur les contractions de l'espace de Hilbert, VI, Calcul functionnel, Acta Sci. Math. Szeged 23 (1962), 130-167.

20. A. E. Taylor, Introduction to functional analysis, Wiley \& Sons, New York, 1958.

Received June 27, 1967. This work is part of a doctoral thesis submitted to the University of Colorado, and was supported by a National Science Foundation Cooperative Graduate Fellowship.

University of New Mexico 



\section{PACIFIC JOURNAL OF MATHEMATICS}

\section{EDITORS}

\author{
H. ROYDEN \\ Stanford University \\ Stanford, California \\ R. R Phelps \\ University of Washington \\ Seattle, Washington 98105
}

\author{
J. Dugundui \\ Department of Mathematics \\ University of Southern California \\ Los Angeles, California 90007
}

RICHARD ARENS

University of California

Los Angeles, California 90024

\section{ASSOCIATE EDITORS}
E. F. BeCKENBACH
B. H. NeUmanN
F. WolF
K. YosidA

\section{SUPPORTING INSTITUTIONS}

\author{
UNIVERSITY OF BRITISH COLUMBIA \\ CALIFORNIA INSTITUTE OF TECHNOLOGY \\ UNIVERSITY OF CALIFORNIA \\ MONTANA STATE UNIVERSITY \\ UNIVERSITY OF NEVADA \\ NEW MEXICO STATE UNIVERSITY \\ OREGON STATE UNIVERSITY \\ UNIVERSITY OF OREGON \\ OSAKA UNIVERSITY \\ UNIVERSITY OF SOUTHERN CALIFORNIA
}

\author{
STANFORD UNIVERSITY \\ UNIVERSITY OF TOKYO \\ UNIVERSITY OF UTAH \\ WASHINGTON STATE UNIVERSITY \\ UNIVERSITY OF WASHINGTON

$* * * * *$
AMERICAN MATHEMATICAL SOCIETY
CHEVRON RESEARCH CORPORATION
TRW SYSTEMS
NAVAL WEAPONS CENTER

The Supporting Institutions listed above contribute to the cost of publication of this Journal, but they are not owners or publishers and have no responsibility for its content or policies.

Mathematical papers intended for publication in the Pacific Journal of Mathematics should be in typed form or offset-reproduced, double spaced with large margins. Underline Greek letters in red, German in green, and script in blue. The first paragraph or two must be capable of being used separately as a synopsis of the entire paper. It should not contain references to the bibliography. Manuscripts, in duplicate if possible, may be sent to any one of the four editors. Please classify according to the scheme of Math. Rev. 36, 1539-1546. All other communications to the editors should be addressed to the managing editor, Richard Arens, University of California, Los Angeles, California, 90024.

50 reprints are provided free for each article; additional copies may be obtained at cost in multiples of 50 .

The Pacific Journal of Mathematics is published monthly. Effective with Volume 16 the price per volume (3 numbers) is $\$ 8.00$; single issues, $\$ 3.00$. Special price for current issues to individual faculty members of supporting institutions and to individual members of the American Mathematical Society: $\$ 4.00$ per volume; single issues $\$ 1.50$. Back numbers are available.

Subscriptions, orders for back numbers, and changes of address should be sent to Pacific Journal of Mathematics, 103 Highland Boulevard, Berkeley, California, 94708.

PUBLISHED BY PACIFIC JOURNAL OF MATHEMATICS, A NON-PROFIT CORPORATION

Printed at Kokusai Bunken Insatsusha (International Academic Printing Co., Ltd.), 7-17. Fujimi 2-chome, Chiyoda-ku, Tokyo, Japan. 


\section{Pacific Journal of Mathematics}

Vol. 28, No. 3

May, 1969

Jon F. Carlson, Automorphisms of groups of similitudes over $F_{3} \ldots \ldots \ldots$

W. Wistar (William) Comfort, Neil Hindman and Stelios A. Negrepontis,

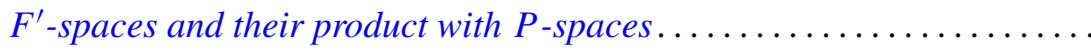

Archie Gail Gibson, Triples of operator-valued functions related to the unit

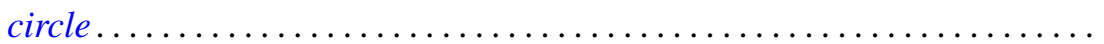

David Saul Gillman, Free curves in $E^{3}$

E. A. Heard and James Howard Wells, An interpolation problem for

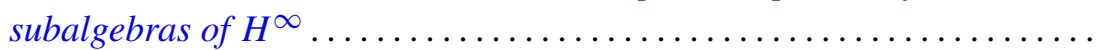

Albert Emerson Hurd, A uniqueness theorem for weak solutions of symmetric

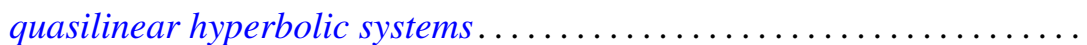

E. W. Johnson and J. P. Lediaev, Representable distributive Noether

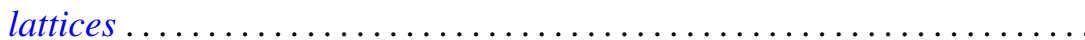

David G. Kendall, Incidence matrices, interval graphs and seriation in

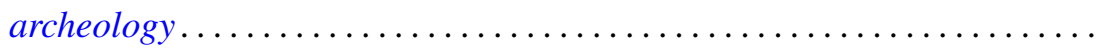
565

Robert Leroy Kruse, On the join of subnormal elements in a lattice ....... 571

D. B. Lahiri, Some restricted partition functions; Congruences modulo 3 .... 575

Norman D. Lane and Kamla Devi Singh, Strong cyclic, parabolic and conical differentiability........................................

William Franklin Lucas, Games with unique solutions that are

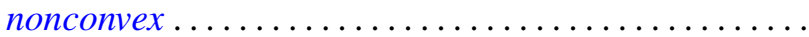

Eugene A. Maier, Representation of real numbers by generalized geometric series.

Daniel Paul Maki, A note on recursively defined orthogonal polynomials ...

Mark Mandelker, $F^{\prime}$-spaces and z-embedded subspaces ...

James R. McLaughlin and Justin Jesse Price, Comparison of Haar series with gaps with trigonometric series

Ernest A. Michael and A. H. Stone, Quotients of the space of irrationals ....

William H. Mills and Neal Zierler, On a conjecture of Golomb ...

J. N. Pandey, An extension of Haimo's form of Hankel convolutions ...

Terence John Reed, On the boundary correspondence of quasiconformal mappings of domains bounded by quasicircles...

Haskell Paul Rosenthal, A characterization of the linear sets satisfying Herz's criterion.

George Thomas Sallee, The maximal set of constant width in a lattice...

I. H. Sheth, On normaloid operators

James D. Stasheff, Torsion in BBSO ...

Billy Joe Thorne, A - P congruences on Baer semigroups.

Robert Breckenridge Warfield, Jr., Purity and algebraic compactness for

modules... 